\title{
The long-time self-diffusivity in concentrated colloidal dispersions
}

\author{
By JOHN F. BRADY \\ Division of Chemistry and Chemical Engineering, California Institute of Technology, Pasadena,
} CA 91125 , USA

(Received 5 July 1993 and in revised form 9 February 1994)

The long-time self-diffusivity in concentrated colloidal dispersions is determined from a consideration of the temporal decay of density fluctuations. For hydrodynamically interacting Brownian particles the long-time self-diffusivity, $D_{\infty}^{s}$, is shown to be expressible as the product of the hydrodynamically determined short-time selfdiffusivity, $D_{0}^{s}(\phi)$, and a contribution that depends on the distortion of the equilibrium structure caused by a diffusing particle. An argument is advanced to show that as maximum packing is approached the long-time self-diffusivity scales as $D_{\infty}^{s}(\phi) \sim$ $D_{0}^{s}(\phi) / g(2 ; \phi)$, where $g(2 ; \phi)$ is the value of the equilibrium radial-distribution function at contact and $\phi$ is the volume fraction of interest. This result predicts that the longtime self-diffusivity vanishes quadratically at random close packing, $\phi_{m} \approx 0.63$, i.e. $D_{\infty}^{s} \sim D_{0}\left(1-\phi / \phi_{m}\right)^{2}$ as $\phi \rightarrow \phi_{m}$, where $D_{0}=k T / 6 \pi \eta a$ is the diffusivity of a single isolated particle of radius $a$ in a fluid of viscosity $\eta$. This scaling occurs because $D_{0}^{s}(\phi)$ vanishes linearly at random close packing and the radial-distribution function at contact diverges as $\left(1-\phi / \phi_{m}\right)^{-1}$. A model is developed to determine the structural deformation for the entire range of volume fractions, and for hard spheres the longtime self-diffusivity can be represented by: $D_{\infty}^{s}(\phi)=D_{0}^{s}(\phi) /[1+2 \phi g(2 ; \phi)]$. This formula is in good agreement with experiment. For particles that interact through hard-spherelike repulsive interparticle forces characterized by a length $b(>a)$, the same formula applies with the short-time self-diffusivity still determined by hydrodynamic interactions at the true or 'hydrodynamic' volume fraction $\phi$, but the structural deformation and equilibrium radial-distribution function are now determined by the 'thermodynamic' volume fraction $\phi_{b}$ based on the length $b$. When $b \gg a$, the long-time self-diffusivity vanishes linearly at random close packing based on the "thermodynamic' volume fraction $\phi_{b m}$. This change in behaviour occurs because the true or 'hydrodynamic' volume fraction is so low that the short-time self-diffusivity is given by its infinite-dilution value $D_{0}$. It is also shown that the temporal transition from short- to long-time diffusive behaviour is inversely proportional to the dynamic viscosity and is a universal function for all volume fractions when time is nondimensionalized by $a^{2} / D_{\infty}^{s}(\phi)$.

\section{Introduction}

The diffusive motion of particles in colloidal dispersions is one of the most fundamental processes occurring in these systems. It can be readily accessed by dynamic light scattering and related to a number of transport and relaxation mechanisms. There are three well-defined diffusive processes occurring in colloidal dispersions: the short-time self-diffusivity, to be denoted by $D_{0}^{s}$, the long-time self- 
diffusivity, $D_{\infty}^{s}$, and the gradient or collective diffusivity, $D^{c} . \dagger$ At infinite dilution all three diffusivities are the same and equal to the Stokes-Einstein diffusivity of a single isolated particle: $D_{0}=k T / 6 \pi \eta a$. Here, $k$ is Boltzmann's constant, $T$ is the absolute temperature, $\eta$ is the viscosity of the continuum suspending fluid, and $a$ is the characteristic particle size.

Although they are the same at infinite dilution, the three diffusivities are quite different at higher concentrations and correspond to distinct physical processes. The short-time self-diffusivity measures the local 'mobility' of a particle on timescales long compared to the momentum relaxation time of a particle, $\tau_{I}=m / 6 \pi \eta a$, but small compared to the time it takes a particle to move a distance a fraction of its size, which is the diffusive or Smoluchowski timescale $\tau_{S}=a^{2} / D_{0}$. Here, $m$ is the mass of the particle, and for particles of density comparable to that of the fluid $\tau_{I} \approx a^{2} / \nu$, where $\nu$ is the kinematic viscosity of the fluid. For colloidal particles as small as $10 \mathrm{~nm} \tau_{S} / \tau_{I} \approx$ $10^{4}$, showing that the particle's momentum has relaxed long before the particle has moved, and that the dynamics are governed by the diffusive Smoluchowski equation. The long-time self-diffusivity, on the other hand, corresponds to motion on times long compared to $a^{2} / D_{0}$, so that a particle has wandered far from its starting point, exchanging places with its neighbours. Finally, the collective or gradient diffusivity corresponds to the diffusivity one would measure resulting from the net flux of all the particles down a macroscopic concentration gradient.

All three diffusivities are measurable in dynamic light scattering experiments. The short- and long-time self-diffusivities can be measured in tracer experiments where a small fraction of the particles are tagged and their motion followed. The relevant quantity measured is the spatial Fourier transform of the tracer density autocorrelation function, $F_{s}(\boldsymbol{k}, t)$, also known as the self-intermediate scattering function, which can be related directly to the scattered light intensity (Berne \& Pecora 1976). Here, $k$ is the wave vector of the scattered light, which corresponds to one over the range of the density fluctuations. For diffusive behaviour the self-intermediate scattering function decays exponentially in time, $F_{s} \sim \exp \left(-k^{2} D t\right)$, from which the diffusivity can be obtained by time differentiation. The short-time self-diffusivity corresponds to the initial slope, while the long-time self-diffusivity is given in the limit $t \rightarrow \infty$. It is only at these two extremes in time that the motion is diffusive. At intermediate times, a diffusing particle distorts its local environment, and the reaction of the distorted structure results in a non-diffusive motion in general. Only at long times when the particle has encountered many independent 'distorted environments' will the central limit theorem apply and the motion be diffusive (Batchelor 1983; Rallison \& Hinch 1986).

In this paper we study the long-time diffusive behaviour in concentrated colloidal dispersions. There have been numerous studies of this problem; only a few of the most recent and relevant can be mentioned: Batchelor (1983), Rallison \& Hinch (1986), Rallison (1988), Pusey (1991), Medina-Noyola (1988), Cohen \& de Schepper (1991), Leegwater \& Szamel (1992), Szamel \& Leegwater (1992) and Cichocki \& Felderhof (1992). The study by Rallison \& Hinch is particularly thorough and careful in its discussion of the limits under which diffusive motion applies. Although there have been a number of studies, no completely satisfactory theory, particularly at high concentrations, has emerged. In this paper we develop a very simple theory that leads to scaling predictions for the long-time self-diffusivity near maximum packing and to

$\dagger$ We are only considering translational diffusion in this paper. There are analogous rotational diffusivities. 
accurate quantitative predictions for all volume fractions. The theory also provides a natural and rigorous connection to rheological behaviour.

As a tracer particle moves in the suspension it deforms its local environment. The distorted structure or 'cage' must then relax to free the tracer to continue its random walk. This distortion and relaxation of the local structure retards the motion of the tracer and is responsible for the long-time self-diffusivity being smaller than the shorttime self-diffusivity. The appropriate timescale for the relaxation of the structure is the particle size squared divided by the long-time self-diffusivity, as particles must move distances of order their size to free the tracer. As the density increases, it is more difficult to distort and relax the structure and the motion slows down, ultimately vanishing at close packing.

Alternatively, one can view (loosely) the diffusing particle as pushing and carrying along its neighbours (or cage) as it moves. This has the effect of increasing the 'size' of the particle, and from the Stokes-Einstein relation the diffusivity decreases as one over the size. At maximum packing the particle becomes 'infinite' in size and the longtime self-diffusivity vanishes. This argument applies whether or not the particles interact hydrodynamically and thus this slowing down can be related to structural (or thermodynamic) properties of the dispersion, particularly to the radial-distribution function at contact, $g(2)$, which is proportional to the effective size of the tracer. Hence, in the absence of hydrodynamic interactions and as the number density approaches maximum packing, we expect $D_{\infty}^{s} \sim D_{0} / g(2)$ - the single-particle diffusivity divided by the new size. For hard-sphere-like repulsive forces the radial-distribution function at contact diverges as $\left(1-n / n_{m}\right)^{-1}$ as $n \rightarrow n_{m}$, and thus we predict that in the absence of hydrodynamic interactions the long-time self-diffusivity vanishes linearly as $n \rightarrow n_{m}$. Here, $n$ is the number density of particles, and the maximum number density, $n_{m}$, is set by the thermodynamic interactions among particles.

With hydrodynamic interactions, the individual particle mobility is no longer the infinite-dilution value $D_{0}$, but rather the average mobility of a particle in the suspension, which is the short-time self-diffusivity $D_{0}^{s}$. Since the increase in effective size is the same with or without hydrodynamics, we expect in general that $D_{\infty}^{s} \sim D_{0}^{s} / g(2)$ as maximum packing is approached. Indeed, we shall see below that such a factorization into hydrodynamic, $D_{0}^{s}$, and structural, $g(2)$, parts is borne out by the analysis and by experiment for all concentrations, not just near maximum packing. We have a very simple physical picture of the diffusion process: the long-time self-diffusivity is proportional to the average diffusivity of an individual particle divided by its characteristic 'size'; the former is set by hydrodynamics and the latter primarily by thermodynamics. For hydrodynamically interacting Brownian hard spheres, the shorttime self-diffusivity vanishes linearly as maximum packing is approached, and thus the long-time self-diffusivity is predicted to vanish quadratically, i.e. $D_{\infty}^{s} \sim D_{0}\left(1-\phi / \phi_{m}\right)^{2}$ as $\phi \rightarrow \phi_{m}$; one factor from $D_{0}^{s}$ and one from $g(2)$. Here, $\phi=\frac{4}{3} \pi n a^{3}$ is the volume fraction of suspended particles and $\phi_{m} \approx 0.63$.

In $\$ 2$ we develop the general equations to describe the temporal decay of density fluctuations, which leads to the dynamic structure factor, and show explicitly how one can readily obtain the short-time and collective diffusivities as equilibrium averages. The long-time self-diffusivity involves the deformation of the structure caused by the diffusing tracer, and in this section we also present the $N$-particle Smoluchowski equation for the perturbed structure. In the next section we reduce the general equations applicable for all time and all wavelengths to the large-wavelength long-time limit appropriate for determining the long-time self-diffusivity. The $N$-particle equations are reduced to an equation for a pair, and a simple scaling argument shows 
that the long-time self-diffusivity factors into the product of the hydrodynamically determined short-time self-diffusivity and a structural term determined by the deformation of the equilibrium distribution caused by the diffusing tracer. To determine the deformed structure the pair-evolution equation must be solved, and, since the motion of a pair is coupled to higher-order distributions, a closure is needed. We close this equation in a self-consistent manner by recognizing that the perturbed structure relaxes with the long-time self-diffusivity, which leads to the scaling prediction that the 'effective size' of the tracer is proportional to the equilibrium radial-distribution function at contact, hence to the prediction that the long-time selfdiffusivity is proportional to the short-time self-diffusivity divided by the equilibrium radial-distribution function at contact. The results of the theory are compared with experiment and computer simulation and are shown to be in good agreement for all volume fractions.

In $\S 4$ we relate the self-diffusivities to the suspension viscosity. The short-time selfdiffusivity is seen to be inversely proportional to the high-frequency dynamic viscosity: $D_{0}^{s} \sim 1 / \eta a \eta_{\infty}^{\prime}$, both being determined by hydrodynamics. From my earlier work on the rheological behaviour of concentrated colloidal dispersions (Brady 1993b), it is shown that the long-time self-diffusivity is inversely proportional to the zero-frequency or steady-shear viscosity: $D_{\infty}^{s} \sim 1 / \eta \eta_{0}^{\prime}$. In this section we also extend the analysis to determine the temporal response as the tracer approaches its long-time diffusive asymptote - so-called memory effects. Perhaps not surprisingly, it is shown that the time Fourier transform of the difference between one over the long- and short-time self-diffusivities, $\left(1 / D_{\infty}^{s}-1 / D_{0}^{s}\right)$, is proportional to the frequency-dependent dynamic viscosity $\eta_{r}(\omega)$. We also predict that the normalized frequency-dependent selfdiffusivity, $\left(1 / D^{s}(\omega)-1 / D_{0}^{s}\right) /\left(1 / D_{\infty}^{s}-1 / D_{0}^{s}\right)$, is a universal function for all $\phi$ of the reduced frequency $\omega a^{2} / D_{\infty}^{s}(\phi)$. Finally, we conclude in $\S 5$ with the observation that this factorization into hydrodynamic and structural parts carries over into other situations, such as the diffusivity in a suspension of rods or in a fixed array.

\section{Density fluctuations and the particle evolution equation}

\subsection{Problem formulation}

The diffusive motion is most conveniently studied by a consideration of the decay of density fluctuations. The particle density at any point $x$ is defined by

$$
n(x, t)=\sum_{\alpha=1}^{N} \delta\left(x-x_{\alpha}(t)\right),
$$

where $\delta$ is the delta function, and $x_{\alpha}(t)$ is the position of particle $\alpha$ at time $t$. The spatial Fourier transform of the density is given by

$$
\hat{n}(\boldsymbol{k}, t)=\int \mathrm{e}^{\mathrm{i} \boldsymbol{k} \cdot \boldsymbol{x}} n(\boldsymbol{x}, t) \mathrm{d} \boldsymbol{x}=\sum_{\alpha=1}^{N} \mathrm{e}^{\mathrm{i} \boldsymbol{k} \cdot \boldsymbol{x}_{\alpha}} .
$$

Since the average density fluctuation is zero, we need to consider the correlation in density fluctuations, specifically, the density fluctuation autocorrelation function, which leads naturally to its spatial Fourier transform, the dynamic structure factor:

$$
\begin{aligned}
F(\boldsymbol{k}, t) & \equiv \frac{1}{N}\langle\hat{n}(\boldsymbol{k}, t) \hat{n}(-\boldsymbol{k}, 0)\rangle-n(2 \pi)^{3} \delta(\boldsymbol{k}) \\
& =\frac{1}{N}\left\langle\sum_{\alpha, \beta}^{N} \mathrm{e}^{\mathrm{i} \boldsymbol{k} \cdot\left(x_{\alpha}(t)-x_{\beta}(0)\right)}\right\rangle-n(2 \pi)^{3} \delta(\boldsymbol{k}),
\end{aligned}
$$


where $n$ is the average number density. The angle brackets in (1) denote an ensemble average over the joint probability $P_{N}\left(x^{N}(t), t ; x^{N}(0), 0\right)$ of particles being at $x^{N}(0)$ at time 0 and at $\boldsymbol{x}^{N}(t)$ at time $t$. Here $\boldsymbol{x}^{N}$ denotes the set of coordinates $\left(\boldsymbol{x}_{1}, \ldots, \boldsymbol{x}_{N}\right)$. Performing the average over the joint probability density, (1) becomes for identical particles

$$
\begin{aligned}
F(k, t) & =\frac{1}{N} \frac{1}{N !} \int \sum_{\alpha, \beta}^{N} \mathrm{e}^{\mathrm{i} k \cdot\left(x_{\alpha}(t)-x_{\beta}(0)\right)} P_{N}\left(x^{N}(t), x^{N}(0)\right) \mathrm{d} x^{N}(t) \mathrm{d} x^{N}(0)-n(2 \pi)^{3} \delta(k) \\
& =\frac{1}{N !} \int\left(\mathrm{e}^{\mathrm{i} k \cdot\left(x_{1}(t)-x_{1}(0)\right)}+(N-1) \mathrm{e}^{\mathrm{i} k \cdot\left(x_{2}(t)-x_{1}(0)\right)}\right) P_{N} \mathrm{~d} x^{N}(t) \mathrm{d} x^{N}(0)-n(2 \pi)^{3} \delta(k) \\
& =F_{s}(k, t)+\frac{N-1}{N !} \int \mathrm{e}^{\mathrm{i} k \cdot\left(x_{2}(t)-x_{1}(0)\right)} P_{N} \mathrm{~d} x^{N}(t) \mathrm{d} x^{N}(0)-n(2 \pi)^{3} \delta(k),
\end{aligned}
$$

where we have introduced the self-intermediate scattering function $F_{s}(k, t)$. The selfdiffusivity of colloidal particles measured in a tracer experiment where a small fraction are tagged is determined by $F_{s}$, and we shall primarily be concerned with this function here.

The joint probability $P_{N}\left(x^{N}(t), x^{N}(0)\right)$ can be expressed in terms of the conditional probability of being at $x^{N}(t)$ given that the particles started at $x^{N}(0)$,

$$
P_{N}\left(x^{N}(t), x^{N}(0)\right)=P_{N}\left(x^{N}(t) \mid x^{N}(0)\right) P_{N}^{0}\left(x^{N}(0)\right),
$$

where $P_{N}^{0}\left(x^{N}(0)\right)$ is the probability of being in the state $x^{N}(0)$. The initial condition on the conditional probability is then

$$
P_{N}\left(x^{N}(t) \mid x^{N}(0)\right)=\delta\left(x^{N}(t)-x^{N}(0)\right) \text { at } t=0 .
$$

From the initial condition we have for the structure factors

and

$$
F_{s}(k, 0)=1 \text {, }
$$

$$
\begin{aligned}
F(k, 0) & =1+\frac{N-1}{N !} \int \mathrm{e}^{i k \cdot\left(x_{2}(0)-x_{1}(0)\right)} P_{N}^{0} \mathrm{~d} x^{N}(0)-n(2 \pi)^{3} \delta(k) \\
& =1+\frac{1}{N} \int \mathrm{e}^{i k \cdot\left(x_{2}(0)-x_{1}(0)\right)} P_{2}^{0} \mathrm{~d} x_{1}(0) \mathrm{d} x_{2}(0)-n(2 \pi)^{3} \delta(k) \\
& =1+n \int \mathrm{e}^{\mathrm{i} k \cdot r}(g(r)-1) \mathrm{d} r \\
& =S_{0}(k),
\end{aligned}
$$

where $S_{0}(k)$, the Fourier transform of the radial-distribution function $g(r)$, is the static structure factor, and $r=x_{2}-x_{1}$.

The conditional probability, to be denoted simply by $P_{N}\left(x^{N}(t) \mid x^{N}(0)\right)=P_{N}$, for the evolution of a suspension of identical spherical particles of radii $a$ subject to Brownian, interparticle and hydrodynamic forces at low Reynolds number satisfies the $\mathrm{N}$-particle Smoluchowski equation:

$$
\frac{\partial P_{N}}{\partial t}+\nabla \cdot j_{N}=0
$$

where the probability flux $\boldsymbol{j}_{N}$ is given by

$$
j_{N}=v P_{N}=R_{F U}^{-1} \cdot\left(F^{P}-k T \nabla \ln P_{N}\right) P_{N} .
$$


In (7) $v$ is the 'velocity' of the particles viewed as an operator, and in writing this we have combined the individual particle velocity vectors (for spherical particles only the translational velocities and the positions of the centres of each particle need be considered) into a single $3 N$ vector $\boldsymbol{v}$. The 'velocity' of a particle is expressible as the product of a mobility times a force. The colloidal interparticle forces are denoted by $F^{P}$, and the Brownian forces are given by $k T$ times the negative gradient with respect to the configuration vector $x$ of the $\log$ of the probability density, $-k T \nabla \ln P_{N}$. The $3 N \times 3 N$ hydrodynamic resistance tensor $\boldsymbol{R}_{F U}$ relates the hydrodynamic force exerted on the particles to their velocities, and its inverse, $\boldsymbol{R}_{F V}^{-1}$, is the $\mathrm{N}$-particle mobility giving the velocities in terms of the forces. From the Stokes-Einstein relation this mobility multiplied by $k T$ is the $N$-particle diffusivity tensor:

$$
\boldsymbol{D}=k T \boldsymbol{R}_{F U}^{-1} \text {. }
$$

With interparticle forces derivable from a potential,

$$
F^{P}=-\nabla V
$$

the equilibrium distribution is given by

$$
j_{N}=0 \text {, }
$$

which, since $D$ is positive definite, requires that

$$
F^{P}=k T \nabla \ln P_{N},
$$

whose solution is the Boltzmann distribution

$$
P_{N}^{e q} \sim \exp (-V / k T),
$$

where the superscript $e q$ denotes equilibrium. For the case of Brownian hard spheres, the potential is infinite if the particles overlap and zero elsewhere, and (8) gives the wellknown hard-sphere distribution. If the initial condition is taken to be the equilibrium one, as we shall do here, then $P_{N}^{0}=P_{N}^{e q}$.

To determine the diffusive behaviour we need to solve (6) for the evolution of the particles and then use this in (2) to determine the dynamic structure factor. This can be simplified by first performing the ensemble average over the initial values $x^{N}(0)$ by introducing (Rallison \& Hinch 1986)

$$
\hat{P}_{N} \equiv \int P_{N}\left(x^{N}(t) \mid x^{N}(0)\right) P_{N}^{0} \mathrm{e}^{-\mathrm{i} k \cdot x_{1}(0)} \mathrm{d} x^{N}(0) .
$$

The self-intermediate scattering function then becomes

$$
F_{s}(k, t)=\frac{1}{N !} \int \hat{P}_{N} \mathrm{e}^{\mathrm{i} k \cdot x_{1}(t)} \mathrm{d} \boldsymbol{x}^{N}(t) .
$$

Similarly, $F(\boldsymbol{k}, t)$ becomes

$$
F(\boldsymbol{k}, t)=F_{s}(\boldsymbol{k}, t)+\frac{N-1}{N !} \int \hat{P}_{N} \mathrm{e}^{\mathrm{i} k \cdot x_{2}(t)} \mathrm{d} x^{N}(t)-n(2 \pi)^{3} \delta(k) .
$$

The probability, $\hat{P}_{N}$, satisfies the same Smoluchowski equation (6), and from (3) the initial condition is

$$
\hat{P}_{N}^{0}=P_{N}^{0} \mathrm{e}^{-\mathrm{i} k \cdot x_{1}}
$$

In order to determine the self-diffusion coefficient we need to consider the temporal 
behaviour of the dynamic structure factor, particularly the time derivatives of $F$ and $F_{s}$. For a simple diffusive process of independent particles $F_{s} \sim \exp \left(-k^{2} D_{0} t\right)$, where $D_{0}=$ $k T / 6 \pi \eta a$ is the diffusion coefficient of an isolated particle. Thus, $\partial F_{s} / \partial t=-k^{2} D_{0} F_{s}$, showing that the diffusion coefficient can be determined from the logarithmic derivative of $F_{s}$. At short times, $t \ll a^{2} / D_{0}$, a particle has hardly moved, and the diffusion coefficient determined from $F_{s}$ is the short-time self-diffusivity. At times long compared to $a^{2} / D_{0}$ but small compared to $1 / k^{2} D_{0}$, a particle has wandered far compared to its size, but still a small distance compared to the lengthscale, $1 / k$, characteristic of the spatial variation of the concentration of tracer particles. Thus in the limit $t \gg a^{2} / D_{0}$, $k a \rightarrow 0$, the time derivative of $F_{s}$ gives the long-time self-diffusivity (Rallison \& Hinch 1986).

The time derivatives of $F_{s}$ and $F$ can be found from (10) and (11) with the help of the Smoluchowski equation to replace $\partial \hat{P}_{N} / \partial t$ with the flux $\hat{J}_{N}$. Integration by parts, noting that the flux at all boundaries is zero, gives

and

$$
\begin{gathered}
\frac{\partial F_{s}}{\partial t}=\dot{F}_{s}(k, t)=\frac{1}{N !} \mathrm{i} k \cdot \int \hat{\jmath}_{1} \mathrm{e}^{i k \cdot x_{1}} \mathrm{~d} \boldsymbol{x}^{N}, \\
\frac{\partial F}{\partial t}=\dot{F}(k, t)=\dot{F}_{s}+\frac{N-1}{N !} \mathrm{i} \boldsymbol{k} \cdot \int \hat{\jmath}_{2} \mathrm{e}^{\mathrm{i} k \cdot x_{2}} \mathrm{~d} \boldsymbol{x}^{N} .
\end{gathered}
$$

\subsection{Short-time self- and collective diffusivities}

The short-time self-diffusivity can be determined from (13) when evaluated at $t=0$. At time 0 the initial distribution is taken to be the equilibrium one and the flux of particle 1 becomes

$$
\hat{\boldsymbol{J}}_{1}=-\sum_{\beta} D_{\mathrm{i} \beta} \cdot \nabla_{\beta}\left[V+\ln \hat{P}_{N}^{0}\right] \hat{P}_{N}^{0},
$$

where $\boldsymbol{D}_{1 \beta}$ is the mobility (multiplied by $k T$ ) giving the velocity of particle 1 due to a force on particle $\beta$, and $V$ has been non-dimensionalized by $k T$. With the initial condition on $\hat{P}_{N}^{0}$ from (12), $\hat{J}_{1}$ becomes

$$
\hat{J}_{1}(0)=D_{11} \cdot \mathrm{i} k \hat{P}_{N}^{0} .
$$

The time derivative of the self-intermediate scattering function becomes

$$
\begin{aligned}
\dot{F}_{s}(k, 0) & =-\frac{1}{N !} \int k \cdot D_{11} \cdot k \hat{P}_{N}^{0} \mathrm{e}^{\mathrm{i} k \cdot x_{1}} \mathrm{~d} x^{N} \\
& =-\frac{1}{N !} \int k \cdot D_{11} \cdot k P_{N}^{0} \mathrm{~d} x^{N} .
\end{aligned}
$$

Noting that $F_{s}(k, 0)=1,(16)$ can be rewritten as

$$
\frac{\dot{F_{s}}(k, 0)}{k^{2} F_{s}(k, 0)}=-D_{0}^{\mathrm{s}}(\phi)
$$

where the short-time self-diffusivity is defined as the average of $D_{11}$ over the equilibrium distribution

$$
D_{0}^{s}(\phi) \equiv \frac{1}{N !} \frac{1}{k^{2}} \int \boldsymbol{k} \cdot \boldsymbol{D}_{11} \cdot \boldsymbol{k} P_{N}^{0} \mathrm{~d} \boldsymbol{x}^{N},
$$

and we have introduced the volume fraction $\phi=\frac{4}{3} \pi n a^{3}$ of the spherical particles. 
In a similar manner, the time derivative of the dynamic structure factor at $t=0$ can be written as

where

$$
\dot{F}(k, 0)=-k^{2} D(k) F(k, 0),
$$

$$
D(k)=\frac{1}{k^{2} S_{0}(k)} \frac{1}{N} \int k \cdot\left(\left\langle\boldsymbol{D}_{11}\right\rangle_{2}^{0}+(N-1)\left\langle\boldsymbol{D}_{21}\right\rangle_{2}^{0} \mathrm{e}^{i k \cdot\left(x_{2}-x_{1}\right)}\right) \cdot k P_{2}^{0} \mathrm{~d} x_{1} \mathrm{~d} x_{2},
$$

and we have introduced the conditionally averaged mobility

$$
\left\langle D_{11}\right\rangle_{2}^{0} \equiv \frac{1}{(N-2) !} \int D_{11}\left(x^{N}\right) P_{N-2 / 2}^{0}\left(x_{3}, \ldots, x_{N} \mid x_{1}, x_{2}\right) \mathrm{d} x_{3}, \ldots, \mathrm{d} x_{N} .
$$

Here, $P_{N-2 / 2}^{0}$ is the conditional probability of particles being at $x_{3}, \ldots, x_{N}$ given that there are particles at $\boldsymbol{x}_{1}$ and $\boldsymbol{x}_{2}$. A similar expression applies for $\left\langle\boldsymbol{D}_{21}\right\rangle_{0}^{2}$, the mobility (multiplied by $k T$ ) of particle 2 due to a force on 1 .

The short-time self-diffusivity is a purely hydrodynamic quantity in that if there were no hydrodynamic interactions among particles then $D_{0}^{s}=D_{0}$, the diffusivity of an isolated particle. The short-time self-diffusivity has been measured experimentally by dynamic light scattering using the above formula (Pusey \& van Megen 1983, Ottewill \& Williams 1987) and determined by Stokesian Dynamics (Phillips, Brady \& Bossis 1988; Ladd 1990); the agreement between experiment and the numerical calculations is excellent. The short-time self-diffusivity has also been calculated analytically by Beenakker \& Mazur (1984); these calculations are in good agreement with experiment up to $\phi \approx 0.4$.

The wavelength-dependent diffusivity $D(k)$ defined in (20) is a combination of both hydrodynamic $(D)$ and thermodynamic $\left(S_{0}(k)\right)$ effects. In the absence of hydrodynamics, however, the result is rather trivial: $D(k)=D_{0} / S_{0}(k)$. The wave-vectordependent diffusivity can be related to the gradient diffusivity of colloidal dispersions, as first pointed out by Russel \& Glendinning (1981), by noting that, since $D_{21}=D_{12}$ for identical particles, the integral in (20) gives the flux of particle 1 due to a force on particle 1 and due to a force of magnitude $\mathrm{e}^{\mathrm{i} k \cdot\left(x_{2}-x_{1}\right)}$ on particle 2 . In the limit $k \rightarrow 0$ the same force is applied to all particles, which corresponds to gradient or collective diffusion, or to sedimentation, showing the close relation between these two situations. The recent experiments of Selim, Al-Naafa \& Jones (1993) show excellent agreement with (20) up to the highest volume fraction measured of 0.25 .

\subsection{Behaviour at arbitrary time}

To determine the temporal response at arbitrary time we introduce the perturbation to the transform of the equilibrium distribution $f_{N}$ :

$$
\hat{P}_{N}=\hat{P}_{N}^{0} F_{s}(k, t)\left[1+f_{N}\right] .
$$

Since the entire problem is linear and proportional to $F_{s}$, it proves convenient to factor out this dependence explicitly. The flux of particle 1 can then be written as

$$
\hat{J}_{1}=\left(\boldsymbol{D}_{11} \hat{P}_{N}^{0} \cdot \mathrm{i} k\left[1+f_{N}\right]-\sum_{\beta} \boldsymbol{D}_{1 \beta} \hat{P}_{N}^{0} \cdot \nabla_{\beta} f_{N}\right) F_{s}
$$

The average perturbation must also be zero,

$$
\frac{1}{N !} \int P_{N}^{0} f_{N} \mathrm{~d} x^{N}=0
$$


The time derivative of the self-intermediate scattering function becomes

$$
\left(\ln F_{s}\right) \equiv \frac{\dot{F_{s}}(\boldsymbol{k}, t)}{F_{s}}=-k^{2} D_{0}^{s}(\phi)+\frac{1}{N !} \mathrm{i} \boldsymbol{k} \cdot \int\left(\boldsymbol{D}_{11} P_{N}^{0} \cdot \mathrm{i} \boldsymbol{k} f_{N}-\sum_{\beta} \boldsymbol{D}_{1 \beta} P_{N}^{0} \cdot \nabla_{\beta} f_{N}\right) \mathrm{d} \boldsymbol{x}^{N}
$$

Similar expressions can be written for $F$ and $\dot{F}$, but we shall only consider the selfintermediate scattering function in the remainder of this work.

Using (22) in the Smoluchowski equation, the equation for the perturbation to the equilibrium structure becomes

$$
\begin{aligned}
P_{N}^{0} \frac{\partial f_{N}}{\partial t}-\sum_{\alpha} \sum_{\beta} \nabla_{\alpha} \cdot D_{\alpha \beta} P_{N}^{0} \cdot \nabla_{\beta} f_{N}+\sum_{\alpha}\left[\boldsymbol{D}_{\alpha 1} \cdot \mathrm{i} \boldsymbol{k}+\mathrm{i} \boldsymbol{k} \cdot \boldsymbol{D}_{1 \alpha}\right] P_{N}^{0} \cdot \nabla_{\alpha} f_{N} \\
+\left(\sum_{\alpha} \boldsymbol{\nabla}_{\alpha} \cdot \boldsymbol{D}_{\alpha \mathbf{1}} P_{N}^{\mathbf{0}} \cdot \mathrm{i} \boldsymbol{k}+\boldsymbol{k} \cdot \boldsymbol{D}_{11} P_{N}^{0} \cdot \boldsymbol{k}+\left(\ln F_{s}\right)\right)\left(1+f_{N}\right)=0
\end{aligned}
$$

with the initial condition

$$
f_{N}\left(x^{N}, 0\right)=0
$$

Note, we have removed the $\mathrm{e}^{-i k \cdot x_{1}}$ from $\hat{P}_{N}^{0}$ in (24)-(27) and thereby introduced the $k$ dependence into the evolution equation for $f_{N}$.

The problem for the temporal decay of the density correlation function now reduces to solving (26) for $f_{N}$ and using this in (25) for $\left(\ln F_{s}\right)$. Note that since $f_{N}(t=0)=0$, the initial value of $\left(\ln F_{s}\right)$ giving the short-time self-diffusivity is trivially recovered. Equation (25) shows that the temporal behaviour of $F_{s}$ (or $\dot{F}_{s}$ ) will be of a memory form giving the current value of $F_{s}(t)$ in terms of its earlier values. The conventional memory from (cf. Ackerson 1978; Cichocki \& Felderhof 1992) can be obtained by writing the perturbation to the equilibrium structure as $\hat{P}_{N}=\hat{P}_{N}^{0}\left(F_{s}+\tilde{f}_{N}\right)$ rather than the form used in (22). The form used in (22) is particularly convenient for extracting the steady perturbation needed to obtain the long-time self-diffusivity. It should also be remarked that (24)-(27) are not restricted to hard spheres, but are valid for any type of interparticle interaction; the explicit reference to the interparticle potential has been replaced by the equilibrium distribution $P_{N}^{0}$. In the next section we consider the smallwavenumber expansion of $\left(\ln F_{s}\right)$ and $f_{N}$ to determine the long-time self-diffusivity.

\section{The long-time self-diffusivity and pair-evolution equation}

\subsection{Small-k expansion}

The long-time self-diffusivity is determined by examining the temporal decay of the self-intermediate scattering function in the dual limits $t \rightarrow \infty, k a \rightarrow 0$. This involves a consideration of the behaviour of $f_{N}$ and $\left(\ln F_{s}\right)$ for small wave vectors and for large times. In the long-wavelength limit we need only determine $\left(\ln F_{s}\right)$ to $O\left(k^{2}\right)$ as this gives the diffusive response. Since $f_{N}$ is of $O(k),(25)$ becomes

$$
\left(\ln F_{s}\right)=-k^{2} D_{0}^{s}-\frac{1}{N !} \mathrm{i} k \cdot \int\left(\sum_{\beta} \boldsymbol{D}_{1 \beta} P_{N}^{0} \cdot \nabla_{\beta} f_{N}\right) \mathrm{d} \boldsymbol{x}^{N}+o\left(k^{2}\right) .
$$

And the evolution equation for $f_{N}$ becomes

$$
P_{N}^{0} \frac{\partial f_{N}}{\partial t}-\sum_{\alpha} \sum_{\beta} \nabla_{\alpha} \cdot \boldsymbol{D}_{\alpha \beta} P_{N}^{0} \cdot \nabla_{\beta} f_{N}=-\sum_{\alpha} \boldsymbol{\nabla}_{\alpha} \cdot \boldsymbol{D}_{\alpha 1} P_{N}^{0} \cdot \mathrm{i} \boldsymbol{k}+o(k) .
$$




\subsection{Pair-evolution equation and the dilute limit}

The evolution equation for the perturbation to the equilibrium pair-distribution function,

$$
f_{2} \equiv \frac{1}{(N-2) !} \int P_{N-2 / 2}^{0} f_{N} \mathrm{~d} x_{N-2}
$$

obtained by integrating (29) over $N-2$ particles is

$$
P_{2}^{0} \frac{\partial f_{2}}{\partial t}-\nabla_{r} \cdot P_{2}^{0}\left\langle\boldsymbol{D}_{r} \cdot \nabla_{r} f_{N}\right\rangle_{2}^{0}-\nabla_{r} \cdot \int\left\langle\left(\boldsymbol{D}_{23}-\boldsymbol{D}_{13}\right) \cdot \nabla_{3} f_{N}\right\rangle_{3}^{0} P_{3}^{0} \mathrm{~d} \boldsymbol{x}_{3}=\nabla_{r} \cdot \frac{1}{2}\left\langle\boldsymbol{D}_{r}\right\rangle_{2}^{0} P_{2}^{0} \cdot \mathrm{i} \boldsymbol{k}
$$

with boundary conditions of no relative flux at the surface of contact of the two particles

$$
\begin{aligned}
\hat{\boldsymbol{r}} \cdot\left[P_{2}^{0}\left\langle\boldsymbol{D}_{r} \cdot \nabla_{r} f_{N}\right\rangle_{2}^{0}+\int\left\langle\left(\boldsymbol{D}_{23}-\boldsymbol{D}_{13}\right) \cdot \nabla_{3} f_{N}\right\rangle_{3}^{0} P_{3}^{0} \mathrm{~d} \boldsymbol{x}_{3}\right] \\
=-\hat{\boldsymbol{r}} \cdot \frac{1}{2}\left\langle\boldsymbol{D}_{r}\right\rangle_{2}^{0} P_{2}^{0} \cdot \mathrm{i} \boldsymbol{k} \text { at } r=2 a,
\end{aligned}
$$

and zero perturbation at large separations,

$$
f_{2} \sim 0 \quad \text { as } r \rightarrow \infty
$$

This outer boundary condition follows from the fact that $f_{2} \sim f_{1}$ as $r \rightarrow \infty$, and a consideration of the single-particle evolution equation will show $f_{1}=0$. The initial condition remains unchanged of no disturbance,

$$
f_{2}(r, 0)=0 .
$$

In writing (31) and (32) we have introduced $\nabla_{r}=\nabla_{2}=-\nabla_{1}$, with $r=x_{2}-x_{1}$ and $\hat{r}=r / r$. The relative diffusivity $D_{r}$ is defined by

$$
D_{r} \equiv D_{22}+D_{11}-D_{21}-D_{12}
$$

Finally, the time derivative of the self-intermediate scattering function becomes

$$
\left(\ln F_{s}\right)=-k^{2} D_{0}^{s}+\frac{1}{N} \mathrm{i} \boldsymbol{k} \cdot \int \frac{1}{2} P_{2}^{0}\left\langle\boldsymbol{D}_{r} \cdot \nabla_{r} f_{N}\right\rangle_{2}^{0} \mathrm{~d} \boldsymbol{r} \mathrm{d} \boldsymbol{x}_{1} .
$$

At long times the self-intermediate scattering function relaxes diffusively, with the characteristic diffusivity being the long-time self-diffusivity at the volume fraction of interest, $D_{\infty}^{s}(\phi)$. Thus, we expect

$$
\left(\ln F_{s}\right) \sim-k^{2} D_{\infty}^{s}(\phi) \text { as } t \rightarrow \infty,
$$

and in (36) only the steady solution for $f_{N}$ is needed.

We first note that in the dilute limit the conditional averages with three particles fixed in (31), (32) and (36) may be neglected, $\left\langle\boldsymbol{D}_{r} \cdot \nabla_{r} f_{N}\right\rangle_{2}^{0}=\left\langle\boldsymbol{D}_{r}\right\rangle_{2}^{0} \cdot \nabla_{r} f_{2}$, and the relative diffusivity becomes that for two particles alone in the fluid. Also, $P_{2}^{0} \approx n^{2}$, and the solution for $f_{2}$ is identical to that given by Batchelor (1983) and Rallison \& Hinch (1986) and gives

$$
\frac{D_{\infty}^{s}(\phi)}{D_{0}}= \begin{cases}1-2 \phi & \text { without hydrodynamics } \\ 1-2.1 \phi & \text { with complete hydrodynamics }\end{cases}
$$




\subsection{Factorization of the long-time self-diffusivity into hydrodynamic and structural parts}

Equation (36) for $\left(\ln F_{s}\right)$ is in dimensional form and the diffusivities under the integral on the right-hand side must be non-dimensionalized. The most obvious choice would be simply to non-dimensionalize $D_{r}$ by the diffusivity of an isolated particle $D_{0}$. However, at large separations between the two fixed particles the conditionally averaged relative diffusivity, $\left\langle\boldsymbol{D}_{r}\right\rangle_{2}^{0}$, asymptotes to $2 D_{0}^{s}(\phi)$, not to $2 D_{0}$. The same applies to the evolution equation for $f_{2}$, which is actually independent of the scale of the diffusivities at steady state. Thus, from (37) and (36) we conclude that

$$
D_{\infty}^{s}(\phi)=D_{0}^{s}(\phi) \times \text { a function of } f_{N}(\phi)
$$

where the function of $f_{N}(\phi)$ is determined by the deformation of the equilibrium structure caused by the diffusing tracer and is independent of the scale of the diffusivity. Although independent of the scale of the diffusivity, the deformation of the structure still has a dependence on hydrodynamic interactions. In prior work on the long-time self-diffusivity (e.g. Medina-Noyola 1988; Cohen \& de Schepper 1991) it has simply been assumed that the factorization displayed in (39) was valid. The argument given here shows that this follows directly from scaling. Note that this scaling is quite general, applying to particles of arbitrary size and shape for any form of interparticle interaction.

\subsection{Closure of the hierarchy and determination of the long-time self-diffusivity}

The above equations are still exact (to $O(k)$ ) and show that the evolution of the pairdistribution function and the time derivative of the self-intermediate scattering function depend on the triplet distribution through $\boldsymbol{D}_{r}, \boldsymbol{\nabla}_{r} f_{N}$, etc., and must be closed in some manner. The simplest closure is to neglect couplings to higher-order terms, which produces the dilute limit referred to above, but this will not be valid at high $\phi$. Furthermore, simply including the correct pair-distribution function or conditionally averaged hydrodynamics but neglecting couplings to the third particle will not necessarily produce an improved approximation. A closure incorporating the effects of all higher-order interactions is needed.

\subsubsection{No hydrodynamic interactions: scaling prediction}

To motivate the closure, we consider first the simplest case of no hydrodynamic interactions, in which case (31), (32) and (36) can be written without approximation as

and

$$
\begin{gathered}
\frac{g(r)}{2 D_{0}} \frac{\partial f_{2}}{\partial t}-\nabla_{r} \cdot g(r)\left\langle\nabla_{r} f_{N}\right\rangle_{2}^{0}=\frac{1}{2} \nabla_{r} g(r) \cdot \mathrm{i} k, \\
\hat{\boldsymbol{r}} \cdot g(r)\left\langle\nabla_{r} f_{N}\right\rangle_{2}^{0}=-\frac{1}{2} g(r) \mathrm{i} \boldsymbol{k} \cdot \hat{r} \quad \text { at } \quad r=2 a, \\
\frac{\left(\ln F_{s}\right)}{D_{0}}=-k^{2} \frac{D_{\infty}^{s}}{D_{0}}=-k^{2}+\mathrm{i} k \cdot \int n g(r)\left\langle\nabla_{r} f_{N}\right\rangle_{2}^{0} \mathrm{~d} r,
\end{gathered}
$$

where we have used $P_{2}^{0}=n^{2} g(r)$, with $g(r)$ the equilibrium radial distribution function. To achieve a closed equation we need to express $\left\langle\boldsymbol{\nabla}_{r} f_{N}\right\rangle_{2}^{\mathbf{0}}$ in terms of $\boldsymbol{\nabla}_{r} f_{2}$, but the differentiation does not commute with the averaging (cf. equation (30), the definition of the conditional average). Indeed, this problem has similarities with the determination of the effective conductivity of a matrix filled with non-conducting particles - a macroscopic concentration gradient of magnitude $i k$ is imposed with no-flux boundary conditions on the particle surfaces. 
To effect the closure we first note that the time derivative of the self-intermediate scattering function can be expressed in terms of the flux of particle 1. That is, (42) can be written as

$$
\frac{\left(\ln F_{s}\right)}{D_{0}}=\mathrm{i} \boldsymbol{k} \cdot \bar{J}_{1},
$$

where the constant flux of particle 1 (scaled by $D_{0}$ ) is

$$
\bar{J}_{1}=\mathrm{i} k+\int n g(r)\left\langle\nabla_{r} f_{N}\right\rangle_{2}^{0} \mathrm{~d} r .
$$

We can replace the forcing vector $i k$ in (40) by the constant-flux vector $\bar{J}_{1}$ to give a selfconsistent set of equations to determine $f_{2}$ :

$$
\frac{g(r)}{2 D_{0}} \frac{\partial f_{2}}{\partial t}-\nabla_{r} \cdot\left[g(r)\left\langle\nabla_{r} f_{N}\right\rangle_{2}^{0}-\frac{1}{2} g(r) \int n g\left(r^{\prime}\right)\left\langle\nabla_{r} f_{N}\right\rangle_{2}^{0} \mathrm{~d} r^{\prime}\right]=\frac{1}{2} \nabla_{r} g(r) \cdot \bar{J}_{1},
$$

along with the correspondingly modified boundary condition at contact. The perturbation to the structure is now forced by the flux $\vec{J}_{1}$ rather than by the concentration gradient of the tracer as represented by $\mathrm{i} k$, and the flux itself is determined self-consistently.

To determine the scaling of the long-time self-diffusivity as maximum packing is approached we note that

$$
\begin{aligned}
\int n g(r)\left\langle\nabla_{r} f_{N}\right\rangle_{2}^{0} \mathrm{~d} r & =\frac{1}{N !} \int P_{N}^{0} \nabla_{2} f_{N} \mathrm{~d} x^{N} \\
& =\frac{1}{N !} \int \nabla_{2}\left(P_{N}^{0} f_{N}\right) \mathrm{d} x^{N}-\frac{1}{N !} \int f_{N} \nabla_{2}\left(\ln P_{N}^{0}\right) P_{N}^{0} \mathrm{~d} x^{N} \\
& =-n g(2) \oint_{r=2 a} f_{2} \hat{r} \mathrm{~d} S-\int n g(r)\left\langle f_{N} \nabla_{r}\left(\ln P_{N}^{0}\right)\right\rangle_{2}^{0} \mathrm{~d} r .
\end{aligned}
$$

Since $f_{2}$ must be linear in the vector $\bar{J}_{1}$ we define

$$
f_{2}(r, t)=f(r, t) \hat{\boldsymbol{r}} \cdot \bar{J}_{1},
$$

and similarly $\left\langle f_{N} \nabla_{r}\left(\ln P_{N}^{0}\right)\right\rangle_{2}^{0}$ will be linear in $\bar{J}_{1}$. Thus, using (46) in (44) and nondimensionalizing $r$ by $a, \bar{J}_{1}$ becomes

$$
\bar{J}_{1}=\frac{\mathrm{i} k}{1+4 \phi g(2) f(2, t)+\phi \int_{2}^{\infty} n g(r)\left\langle f_{N} \mathrm{~d} \ln P_{N}^{0} / \mathrm{d} r\right\rangle_{2}^{0} r^{2} \mathrm{~d} r} .
$$

As maximum packing is approached the equilibrium radial distribution function at contact diverges as (Woodcock 1981)

$$
g(2 ; \phi) \sim 1.2\left(1-\phi / \phi_{m}\right)^{-1} \text { as } \phi \rightarrow \phi_{m},
$$

with $\phi_{m} \approx 0.63$. Since the integral in (48) remains $O(1)$ as $\phi \rightarrow \phi_{m}$, as does $f_{2}$, the dominant contribution to $\bar{J}_{1}$, and therefore to $D_{\infty}^{s}$, comes from $g(2) f(2, t)$, and we predict the scaling

$$
D_{\infty}^{s} \sim D_{0} / g(2) \sim D_{0}\left(1-\phi / \phi_{m}\right) \quad \text { as } \quad \phi \rightarrow \phi_{m} .
$$

The long-time self-diffusivity vanishes linearly at maximum packing. 


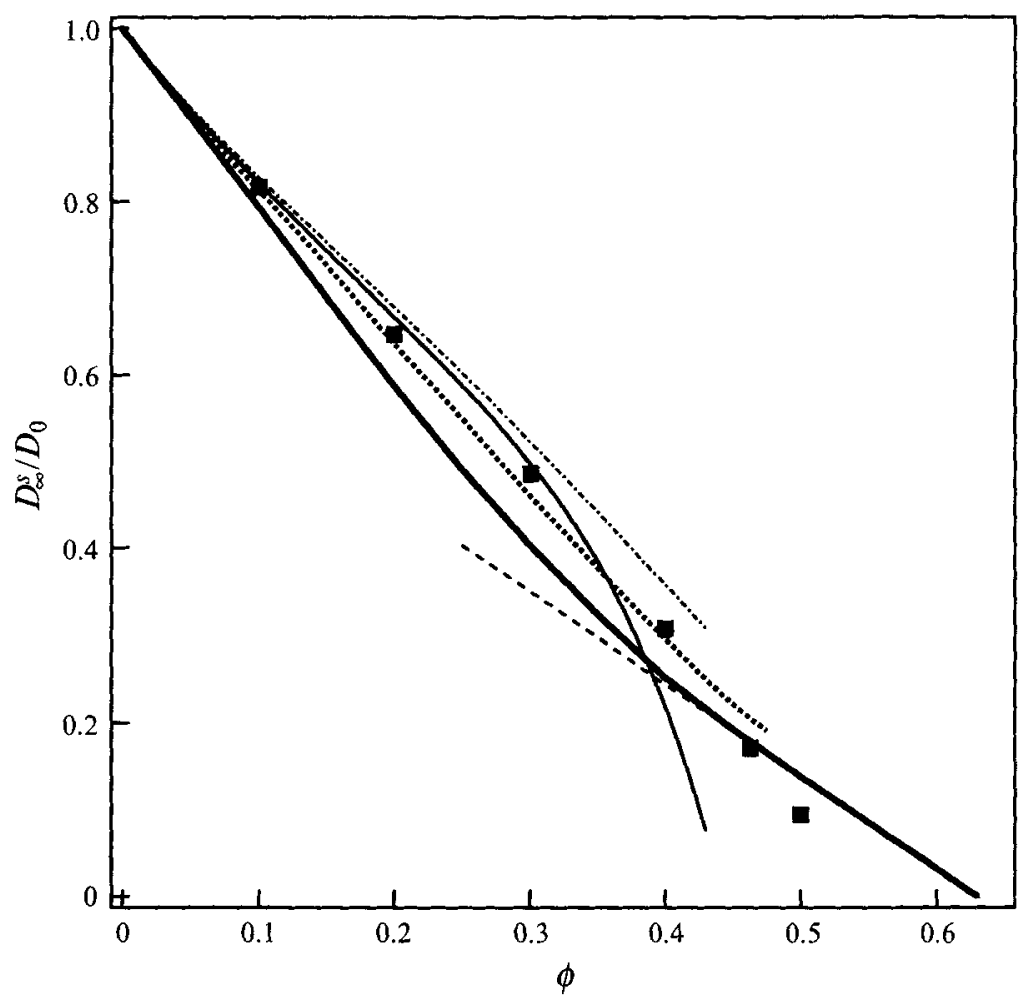

FIGURE 1. Comparison of the theoretical prediction for the long-time self-diffusivity in the absence of hydrodynamic interactions, equation (51), solid line, with the numerical simulation results of Cichocki \& Hinsen $(1990,1992)$ ( $\mathbf{1})$. The dashed line is the asymptotic prediction as random close packing, $\phi_{m}=0.63, \phi_{m}=0.63$, is approached: $D_{\infty}^{s} \sim 1.5\left(1-\phi / \phi_{m}\right)$. The thin solid line and the dot-dashed line are approximate closures discussed in the text. The dotted line is the prediction of Szamel \& Leegwater (1992). $D_{\infty}^{s}(\phi)$ has been non-dimensionalized by the Stokes-Einstein diffusivity $D_{0}=k T / 6 \pi \eta a$.

\subsubsection{No hydrodynamic interactions: estimate for all $\phi$}

To determine the precise value of the long-time self-diffusivity we must solve the pair-evolution equation (45) for $f_{2}$, which is now an integro-differential equation with a 'renormalized' pair diffusivity. The appropriate timescale is seen to be $a^{2} g(2) / D_{0}$ or from (49) $a^{2} / D_{\infty}^{s}$. Physically, the relaxation of the deformed structure and the approach to steady state is with the long-time self-diffusivity as the tracer must move a distance of order its size. Note that only the value of $f_{2}$ at contact is needed for the asymptotic behaviour, and thus a simple estimation of this value is all that is required. We estimate $f_{2}$ at contact at steady state by its dilute value

$$
f(2)=\frac{1}{2}
$$

and the long-time self-diffusivity for all $\phi$ is approximately given by

$$
D_{\infty}^{s}(\phi)=D_{0}[1+2 \phi g(2 ; \phi)]^{-1} \text {. }
$$

This expression correctly reproduces the dilute limit (38) and has the proper scaling at maximum packing.

In figure 1 we show a comparison of (51) with the Brownian dynamics simulations of Cichocki \& Hinsen $(1990,1992)$. In these simulations the particles interacted through hard-sphere repulsive forces, but there were no hydrodynamic interactions 
(i.e. $\boldsymbol{D}_{\alpha \alpha}=D_{0} \boldsymbol{I}$ and $\boldsymbol{D}_{\alpha \beta}=0$ in the Smoluchowski equation). The values of the equilibrium radial-distribution function at contact were found from the CarnahanStarling (Carnahan \& Starling 1969) equation of state for $\phi<0.5$ and from the molecular dynamics simulations of Woodcock (1981) for $\phi>0.5$. The prediction is in reasonable agreement with the simulation results, with what appears to be a slight over estimation of the long-time self-diffusivity at large $\phi$.

To test the sensitivity to the estimate of $f_{2}(2)$, in figure 1 we show two other approximate solutions. In the first - the thin solid line - (45) was solved numerically by approximating $\left\langle\nabla_{r} f_{N}\right\rangle_{2}^{0}$ with $\nabla_{r} f_{2}$ and using the Percus-Yevick equation for $g(r)$. The full expression (48) was used for $D_{\infty}^{s}$, but the dominant contribution was always $g(2) f(2)$. This approximation works well for dilute and moderate $\phi$, but drastically under-predicts the long-time self-diffusivity for high $\phi$ owing to an over-estimation of $f(2)$. In the second approach - the dot-dashed line - the renormalized pair diffusivity when multiplied by $g(r)$ was approximated as a constant, i.e. $\nabla^{2} f_{2}=-\frac{1}{2} \nabla g \cdot \bar{J}_{1}$ was solved. This approximation works rather well, although now slightly over-estimating the long-time self-diffusivity. For a closure to work well it is essential that $f(2)$ remain near the dilute value of $\frac{1}{2}$.

Also shown in figure 1 is the theoretical prediction of Szamel \& Leegwater (1992) for the long-time self-diffusivity in the absence of hydrodynamic interactions. Szamel \& Leegwater (1992) arrived at an expression similar to (48) for the flux of the tracer particle and a similar 'renormalized' pair-evolution equation. In their approach they made an appeal to the fluctuation-dissipation theorem in order to allow them to compute the tracer particle's drag coefficient rather than its mobility and then used this in a frequency- and wavelength-dependent Stokes-Einstein relation. This is entirely equivalent to replacing $\mathrm{i} k$ by $\bar{J}_{1}$ as done here, although we do not need to appeal to any thermodynamic arguments. In most prior studies of the long-time self-diffusivity (e.g. Medina-Noyola 1988; Hess \& Klein 1983) the drag coefficient rather than the mobility was also calculated and the fluctuation-dissipation theorem used to relate it to the diffusivity. Szamel \& Leegwater's improved estimate of $D_{\infty}^{s}$, as their better agreement with the numerical results would seem to indicate, comes from determining $f_{2}$ by considering three-particle interactions and closing the hierarchy by a decoupling approximation, rather than using the dilute estimate for $f_{2}(2)$ as we have done here. They did not, however, note the asymptotic scaling relation near maximum packing.

\subsubsection{No hydrodynamic interactions: repulsive interparticle forces}

Before moving on to consider hydrodynamic interactions, it is instructive to consider particles that interact through hard-sphere-like repulsive forces characterized by a length $b$ greater than $a$. All the considerations leading up to (48) are still valid, but now we find that $g(2) \equiv 0$ because there are no particles precisely at contact at $r=2 a$. However, there is now a singular force at $2 b$ and thus $\nabla_{r} \ln P_{N}^{0}$ will have a delta function at contact at $2 b$ and the volume integral in (46), or in (48), will project out precisely $g$ and $f$ at contact at $2 b$. The long-time self-diffusivity then becomes

$$
D_{\infty}^{s}(\phi)=D_{0}\left[1+2 \phi_{b} g\left(2 ; \phi_{b}\right)\right]^{-1}, \quad b>a,
$$

where $\phi_{b}=\frac{4}{3} \pi n b^{3}$ is the 'thermodynamic' volume fraction based on the length $b$.

\subsubsection{Hydrodynamic interactions}

We would now like to show that with hydrodynamic interactions the long-time selfdiffusivity still scales as $D_{0}^{s}(\phi) / g(2 ; \phi)$ as $\phi \rightarrow \phi_{m}$ and that $(51)$, with $D_{0}$ replaced by $D_{0}^{s}$, a function of $\phi$, is still an accurate estimate over the entire range of $\phi$. Physically, one 
should expect a similar scaling since the effective size of the tracer, as measured by $g(2)$, is the same with or without hydrodynamic interactions.

First we note that $i k$ can be replaced by $\bar{J}_{1}$ as before to obtain the renormalized pairevolution equation

$$
\begin{aligned}
& \frac{g}{D_{0}^{s}} \frac{\partial f_{2}}{\partial t}-\nabla_{r} \cdot\left[g(r)\left\langle\overline{\boldsymbol{D}}_{r} \cdot \nabla_{r} f_{N}\right\rangle_{2}^{\mathbf{0}}-\frac{1}{2} g(r)\left\langle\overline{\boldsymbol{D}}_{r}\right\rangle_{2}^{\mathbf{0}} \cdot \int \frac{1}{2} n g\left(r^{\prime}\right)\left\langle\overline{\boldsymbol{D}}_{r} \cdot \nabla_{r^{\prime}} f_{N}\right\rangle_{2}^{\mathbf{0}} \mathrm{d} \boldsymbol{r}^{\prime}\right] \\
& -\nabla_{r} \cdot \frac{1}{n}\left[\int\left\langle\left(\overline{\boldsymbol{D}}_{23}-\overline{\boldsymbol{D}}_{13}\right) \cdot \nabla_{3} f_{N}\right\rangle_{3}^{0} P_{3}^{0} \mathrm{~d} \boldsymbol{x}_{3}\right]=\nabla_{r} \cdot \frac{1}{2} g(r)\left\langle\overline{\boldsymbol{D}}_{r}\right\rangle_{2}^{0} \cdot \bar{J}_{1},
\end{aligned}
$$

with the corresponding no-flux boundary condition at contact. The overbars denote a normalization of the diffusivities by $D_{0}^{s}(\phi)$ as discussed in $\S 3.3$. The constant flux $\bar{J}_{1}$ becomes

$$
\overline{J_{1}}=\mathrm{i} k+\int \frac{1}{2} n g(r)\left\langle\overline{\boldsymbol{D}}_{r} \cdot \nabla_{r} f_{N}\right\rangle_{2}^{0} \mathrm{~d} \boldsymbol{r}
$$

and the integral will again be proportional to $\bar{J}_{1}$. Integrating by parts as before we have

$$
\begin{aligned}
\int \frac{1}{2} n g(r)\left\langle\overline{\boldsymbol{D}}_{r} \cdot \nabla_{r} f_{N}\right\rangle_{2}^{0} \mathrm{~d} r=-2 \phi g(2) \oint_{r=2 a}\left\langle\hat{\boldsymbol{r}} \cdot \overline{\boldsymbol{D}}_{r} f_{N}\right\rangle_{2}^{0} \mathrm{~d} S \\
\quad-\int \frac{1}{2} n g(r)\left\langle f_{N} \overline{\boldsymbol{D}}_{r} \cdot\left(\boldsymbol{\nabla}_{r} \ln \boldsymbol{P}_{N}^{0}\right)\right\rangle_{2}^{0} \mathrm{~d} \boldsymbol{r}-\int \frac{1}{2} n g(r)\left\langle f_{N}\left(\boldsymbol{\nabla}_{r} \cdot \overline{\boldsymbol{D}}_{r}\right)\right\rangle_{2}^{0} \mathrm{~d} \boldsymbol{r} .
\end{aligned}
$$

The first integral on the right-hand side of (55), which gave the contact value of $g(r)$ without hydrodynamics, is zero with complete hydrodynamics because $\hat{r} \cdot \overline{\boldsymbol{D}}_{r}$ is the relative radial mobility of two particles at contact, which is zero. The contact value of $g(r)$ comes from the third integral on the right-hand side because as maximum packing is approached $\nabla_{r} \cdot \bar{D}_{r}$ is singular at contact. For hard spheres $\bar{D}_{r} \cdot\left(\nabla_{r} \ln P_{N}^{0}\right) \equiv 0$ because the flux of each particle at equilibrium is zero, and it is $\nabla_{r} \cdot \bar{D}_{r}$ that ensures that the proper equilibrium distribution is obtained. With complete hydrodynamics no interparticle potential is needed to achieve hard-sphere behaviour; hydrodynamics alone are sufficient (Brady 1993a). Physically, the singularity in $\boldsymbol{\nabla}_{r} \cdot \overline{\boldsymbol{D}}_{r}$ arises because the variation in the relative mobility of two particles is small at close packing for all separations not near contact because the relative motion is resisted by the effective viscosity of the suspension, not by the viscosity of the solvent. Near maximum packing the effective viscosity, which in this case will be the high-frequency dynamic viscosity, diverges. For two particles in contact on the other hand, the relative motion is resisted by the viscosity of the solvent, not that of the suspension, and this results in $\nabla_{r} \cdot \bar{D}_{r}$ having a delta function at contact. (See Bossis, Brady \& Mathis 1988 for values of $\left\langle\boldsymbol{D}_{r}\right\rangle_{2}^{0}$.) There is a compensation between complete hydrodynamics and hard-sphere behaviour without hydrodynamics (Brady 1993a). This delta function in the last integral will project our precisely the same factor of $g(2) f_{2}(2, t)$ as in the case without hydrodynamics. As before this term is also the dominant contribution near maximum packing and therefore we have the scaling

$$
D_{\infty}^{s}(\phi) \sim D_{0}^{s}(\phi) / g(2 ; \phi) \quad \text { as } \quad \phi \rightarrow \phi_{m} .
$$

It remains only to estimate the value of $f_{2}(2)$ from the solution of $(53)$. We shall use 


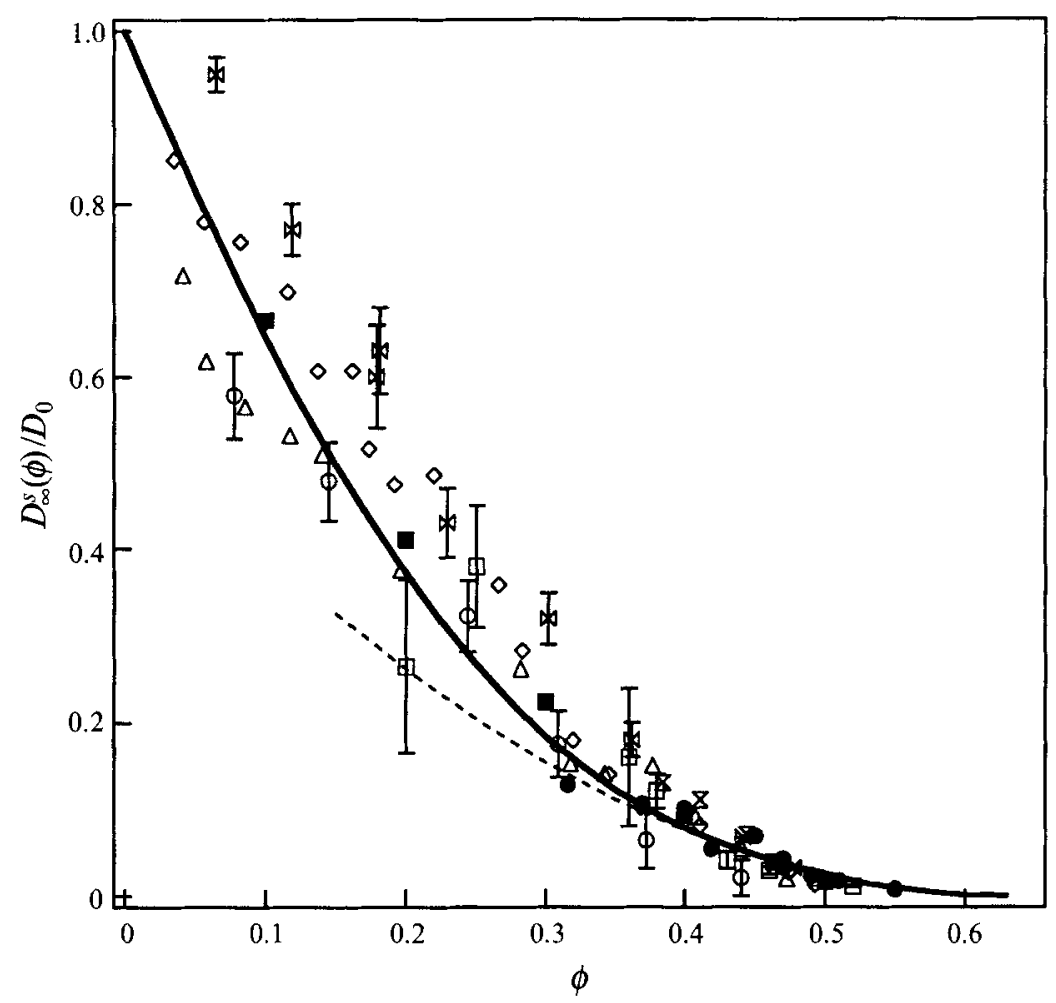

FIGURE 2. Comparison of the theoretical prediction, equation (57), solid line, for the long-time selfdiffusivity of Brownian hard spheres with various experimental results and the Stokesian dynamics simulations of Phung (1993). The dashed line is the asymptotic prediction as random close packing, $\phi_{m}=0.63$, is approached: $D_{\infty}^{s} \sim 0.562\left(1-\phi / \phi_{m}\right)^{2} . D_{\infty}^{s}(\phi)$ has been non-dimensionalized by the Stokes-Einstein diffusivity $D_{0}=k T / 6 \pi \eta a$. The solid squares are the long-time self-diffusivities in the absence of hydrodynamic interactions determined from computer simulations by Cichocki \& Hinsen $(1990,1992)$ multiplied by the hydrodynamically determined short-time self-diffusivities (Phillips $e t$ al. 1988; Ladd 1990). $₫$, van Blaaderen, et al. (1992); $\diamond$, van Megen \& Underwood (1989); O, van Veluwen \& Lekkerkerker (1988); $\triangle$, Ottewill \& Williams (1987); $Z$, van Megen, Underwood \& Snook (1986); $\square$, Kops-Werkhoven \& Fijnaut (1982); O, Phung (1993); $N=27 ; P e=0.01$.

the same simple dilute estimate as we did without hydrodynamics to approximate $D_{\infty}^{s}$ as

$$
D_{\infty}^{s}(\phi)=D_{0}^{s}(\phi)[1+2 \phi g(2 ; \phi)]^{-1},
$$

for the entire range of $\phi \dagger$.

In figure 2 we compare our prediction, (57), for the long-time self-diffusivity with the experiments of Kops-Werkhoven \& Fijnaut (1982), van Megen, Underwood \& Snook (1986), Ottewill \& Williams (1987), van Veluwen \& Lekkerkerker (1988), van Megen \& Underwood (1989), van Blaaderen et al. (1992) and the Stokesian dynamics simulations of Phung (1993). (Note that the simulation results are for 27 particles in steady simple shear at Péclet number $P e=\dot{\gamma} a^{2} / D_{0}=0.01$, where $\dot{\gamma}$ is the magnitude of the shear rate.) The agreement is seen to be excellent for all $\phi$. In figure 3 we show the same data but on a log-linear plot to amplify the data near close packing. In making these plots we have used the short-time self-diffusivities determined by Stokesian

† This expression, of course, does not correctly reproduce Batchelor's dilute- $\phi$ limit (although a complete solution of the dilute two-body problem would), and is only intended as a simple analytical estimate for all $\phi$ with the proper asymptotic form at maximum packing. 


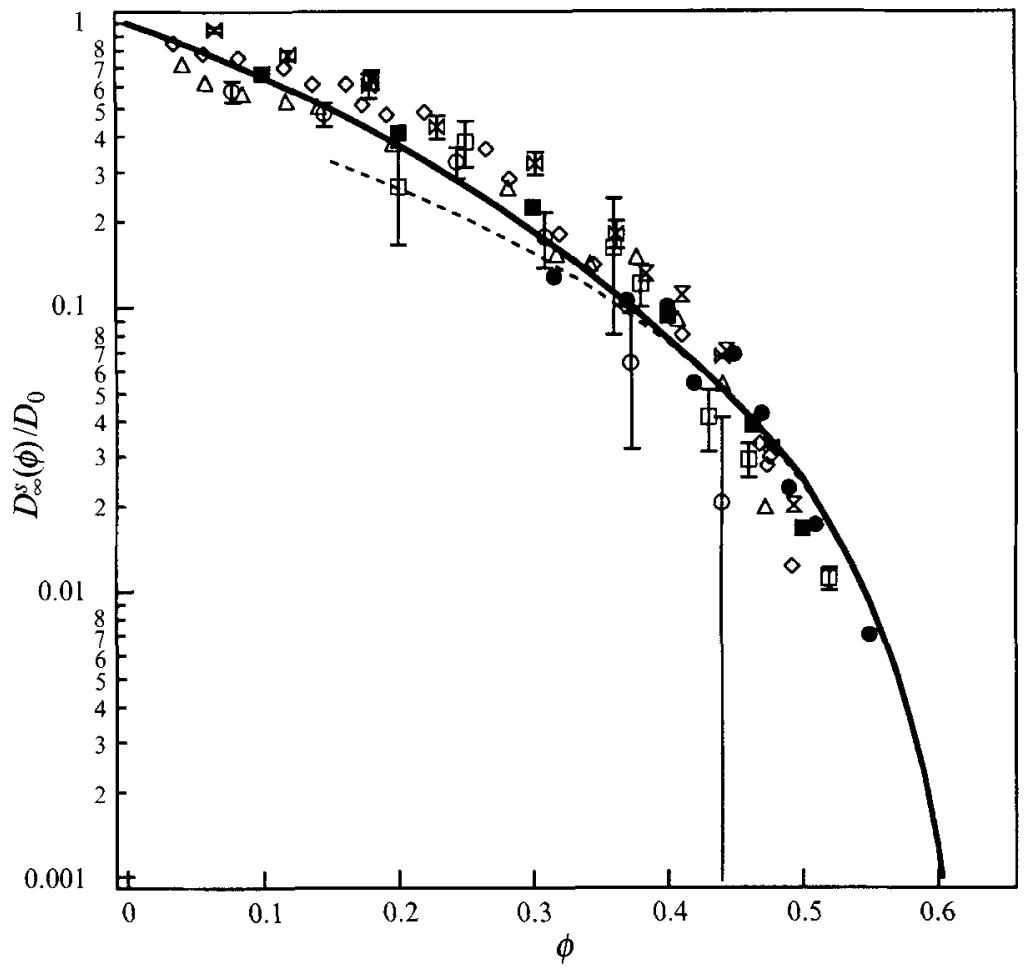

FIGURE 3. The same as figure 2 but shown on a log-linear plot to amplify the region of small diffusivity near close packing.

Dynamics simulations (Phillips et al. 1988; Ladd 1990), which are in good agreement with the experiment for all $\phi$.

The theory predicts that the long-time self-diffusivity vanishes quadratically at random close packing $\phi_{m} \approx 0.63$ :

$$
D_{\infty}^{s} / D_{0} \sim D_{0}^{s}(\phi) / 2 \phi g(2 ; \phi) \sim 0.562\left(1-\phi / \phi_{m}\right)^{2} \text { as } \phi \rightarrow \phi_{m} .
$$

This behaviour arises because the short-time self-diffusivity $D_{0}^{s}(\phi) \sim 0.85\left(1-\phi / \phi_{m}\right)$ as random close packing is approached (Brady 1993b; Phung 1993) and the radialdistribution function at contact diverges as $g(2 ; \phi) \sim 1.2\left(1-\phi / \phi_{m}\right)^{-1}$ as $\phi \rightarrow \phi_{m}$ (Woodcock 1981). The experimental data appear to agree with this prediction, although the formula tends to over predict the diffusivity at high $\phi$. Recall that the hard-sphere system undergoes a phase transition to an FCC solid at $\phi \approx 0.5$, and we have assumed that the dispersion remains random all the way up to close packing. It is quite possible that the experiments are showing the onset of the phase transition for $\phi$ near 0.5 , with a corresponding decrease in the long-time self-diffusivity.

Although the simple prediction in (57) is in reasonable agreement with experiment, we can also test the factorization of the long-time self-diffusivity into hydrodynamic and thermodynamic parts, as predicted by (39), directly by multiplying the long-time self-diffusivities obtained in the simulations of Cichocki \& Hinsen (1992) for hard spheres in the absence of hydrodynamics by the hydrodynamically determined shorttime self-diffusivity $D_{0}^{s}$. The results of doing this are plotted as the solid squares in figures 2 and 3 , showing that this factorization is borne out by the experimental data.

The reason why this factorization works is that the number of neighbours that the moving tracer particle must carry with it, i.e. its cage, is the same with and without hydrodynamics. The size of the cage is directly proportional to the number of near neighbours, which is measured by the equilibrium radial-distribution function at 
contact. This cage increases the size of the tracer particle, and from the Stokes-Einstein relation the diffusivity is proportional to the mobility of an individual particle divided by this increase in size. Hydrodynamics affects the mobility of the individual particle; the average mobility of a particle is the short-time self-diffusivity. Hence, we expect on physical grounds that $D_{\infty}^{s} \sim D_{0}^{s} / g(2)$, at least for large $\phi$, which is precisely what this theory predicts.

\section{Memory effects and the relation to the dynamic viscosity}

In the previous section we showed that the slowing down of the long-time selfdiffusivity could be interpreted from the Stokes-Einstein relation as due to the increased size of the tracer. An alternative perspective is to use the Stokes-Einstein equation to relate the self-diffusivity to the effective viscosity of the suspension; that is, $D \sim k T / a \eta_{\text {eff }}$. The question is, what is the appropriate effective viscosity to use?

When a colloidal dispersion is subjected to a small-amplitude (so that the Péclet number, $P e=\dot{\gamma} a^{2} / D_{0} \ll 1$, where $\dot{\gamma}=|\boldsymbol{E}|$ ) oscillatory shear of the form $\boldsymbol{E} \mathrm{e}^{i \omega t}$, where $\boldsymbol{E}$ is the rate of strain of the imposed shear flow and $\omega$ is the frequency, the dispersion responds viscoelastically and can be characterized by a dynamic viscosity with real and imaginary parts (van der Werff et al. 1989; Brady 1993b)

$$
\eta_{r}(\omega)=\eta^{\prime}(\omega)-\mathrm{i} \eta^{\prime \prime}(\omega),
$$

where $\eta_{r}$ is the viscosity of the suspension divided by that of the solvent. The real part corresponds to dissipation and the imaginary part to elasticity.

At high frequencies the suspension is not perturbed from its equilibrium state, and therefore the dynamic viscosity is purely real and defines the high-frequency dynamic viscosity

$$
\eta_{\infty}^{\prime} \equiv \eta^{\prime}(\omega \rightarrow \infty)=\eta_{r}(\omega \rightarrow \infty) \text {. }
$$

The high-frequency dynamic viscosity is a purely hydrodynamic quantity caused by the fact that the rigid particles do not deform as fluid elements. Since the stucture is not perturbed, the averaging implied is over the equilibrium distribution. For small volume fractions $\eta_{\infty}^{\prime} \sim 1+\frac{5}{2} \phi$; the 1 is from the solvent and the $\frac{5}{2} \phi$ is the Einstein viscosity correction. At high concentrations $\eta_{\infty}^{\prime} \sim\left(1-\phi / \phi_{m}\right)^{-1}$ due to the lubrication singularities in the hydrodynamic interactions among particles. The short-time selfdiffusivity is also purely hydrodynamic in origin, defined as an equilibrium average and has the inverse scaling as $\phi \rightarrow \phi_{m}$. Thus, at least at high $\phi$, the Stokes-Einstein relation gives

$$
D_{0}^{s}(\phi) \sim \frac{k T}{\eta a \eta_{\infty}^{\prime}(\phi)}
$$

The short-time self-diffusivity scales as one over the high-frequency dynamic viscosity. That there should be a relationship between the short-time self-diffusivity and the highfrequency dynamic viscosity was also explored theoretically by Beenakker \& Mazur (1984) and Beenakker (1984).

As we saw in the previous section, the long-time self-diffusivity is not simply an equilibrium average, but involves the steady deformation of the structure by the diffusing tracer. This deformation is similar to the deformation caused by a steady shearing motion, and thus we should expect the long-time self-diffusivity to be related to the zero-frequency or steady-shear viscosity

$$
\eta_{0}^{\prime} \equiv \eta^{\prime}(\omega \rightarrow 0)=\eta_{r}(\omega \rightarrow 0)
$$


In my earlier paper on the rheological behaviour of colloidal dispersions (Brady $1993 \mathrm{~b}$ ), I showed that the steady-shear viscosity was given to a very good approximation by

$$
\eta_{0}^{\prime}=\eta_{\infty}^{\prime}+\frac{12}{5} \phi^{2} \frac{g(2 ; \phi)}{D_{0}^{s}(\phi)}
$$

where the last term is a contribution from the deformation of the equilibrium structure by the shearing motion. The form of the last term, $g(2) / D_{0}^{s}$, is an asymptotic scaling relation as maximum packing is approached. Equation (61) is in excellent agreement with experiment for all $\phi$, and shows that the steady-shear viscosity diverges at random close packing as $\eta_{0}^{\prime} \sim\left(1-\phi / \phi_{m}\right)^{-2}$; the divergence coming from the dominant last term. From (57) for the long-time self-diffusivity we see that $D_{\infty}^{s}$ and $\eta_{0}^{\prime}$ are indeed related, and that at high $\phi$ we predict the precise relation

$$
D_{\infty}^{s}(\phi)=\frac{6}{5} \phi \frac{k T}{6 \pi \eta a} \frac{1}{\eta_{0}^{\prime}(\phi)} \text { as } \phi \rightarrow \phi_{m} .
$$

The long-time self-diffusivity is inversely proportional to the zero-frequency or steadyshear viscosity. The coefficient $\frac{6}{\overline{5}} \phi$ comes from using the simple dilute estimate for $f_{2}$ at contact and from an analogous simple estimate in shearing motion. Thus, while the coefficient in (62) may not be correct, the scaling relation is valid at high $\phi$.

The close relationship between the self-diffusivities and the dynamic viscosities at the two extremes in frequency suggests that this relationship may carry over to all frequencies. To address this issue we need to consider the temporal behaviour of the diffusive motion, or how the particle goes from the short- to the long-time selfdiffusivity. This leads to so-called memory effects in diffusion.

In the previous section (equation (48)) we saw that the long-time self-diffusivity can be written approximately as

$$
D_{\infty}^{s}(\phi)=D_{0}^{s}(\phi)[1+4 \phi g(2, \phi) f(2, t)]^{-1},
$$

where $f(2, t)$ is the perturbation to the equilibrium pair-distribution function at contact. Only the steady solution for $f$ was needed to obtain the long-time self-diffusivity, but we can use this equation to capture the temporal approach to this limit. (We are only interested in the diffusive behaviour, so the small- $k$ expansion used is still valid.) The form of (63) suggests that the proper way to examine the temporal response is through $\left(1 / D_{\infty}^{s}-1 / D_{0}^{8}\right)$ :

$$
\frac{1}{D_{\infty}^{s}}-\frac{1}{D_{0}^{s}}=4 \phi \frac{g(2 ; \phi)}{D_{0}^{s}(\phi)} f(2, t),
$$

or, upon introducing the Fourier transform in time, denoted by *,

$$
\frac{\tilde{1}}{D_{\infty}^{s}}-\frac{\tilde{1}}{D_{0}^{s}}=4 \phi \frac{g(2 ; \phi)}{D_{0}^{s}(\phi)} \tilde{f}(2, \omega) .
$$

Using the dilute approximation for $f_{2}$, the equation for the transform $\tilde{f}$ is

$$
\begin{gathered}
\frac{1}{r^{2}} \frac{\mathrm{d}}{\mathrm{d} r}\left(r^{2} \frac{\mathrm{d} \tilde{f}}{\mathrm{~d} r}\right)-2 \frac{\tilde{f}}{r^{2}}-\mathrm{i} \alpha \tilde{f}=0, \\
\tilde{f} \sim 0 \text { as } \quad r \rightarrow \infty, \\
\frac{\mathrm{d} \tilde{f}}{\mathrm{~d} r}=-\frac{1}{2}\left(\pi \delta(\omega)+\frac{1}{\mathrm{i} \omega}\right) \text { at } \quad r=2,
\end{gathered}
$$


where $\alpha=\omega a^{2} / 2 D_{\infty}^{s}$, and the initial condition has been replaced by the Heaviside forcing at the boundary $r=2 ;(\pi \delta(\omega)+1 / \mathrm{i} \omega)$ is the Fourier transform of the Heaviside function.

Equation ( $66 a$ ) is Bessel's equation with solution

$$
\left.\tilde{f}(r, \omega)=\frac{2}{r^{2}} \exp \left[(i \alpha)^{\frac{1}{2}}\right)(2-r)\right]\left[\frac{1+\frac{1}{2} z_{0} r}{1+z_{0}+\frac{1}{2} z_{0}^{2}}\right]\left(\pi \delta(\omega)+\frac{1}{i \omega}\right),
$$

where

$$
z_{0}=2(\mathrm{i} \alpha)^{\frac{1}{2}} .
$$

At contact

$$
\tilde{f}(2, \omega)=\frac{1}{2}\left[\frac{1+z_{0}}{1+z_{0}+\frac{1}{2} z_{0}^{2}}\right]\left(\pi \delta(\omega)+\frac{1}{\mathrm{i} \omega}\right) .
$$

In my theory of rheological behaviour (Brady 1993b), I showed that for arbitrary frequency the difference between $\eta_{0}^{\prime}$ and $\eta_{\infty}^{\prime}$, i.e. (61), could be written as

$$
\eta_{0}^{\prime}-\eta_{\infty}^{\prime}=\frac{9}{5} \phi^{2} \frac{g(2 ; \phi)}{D_{0}^{s}(\phi)} \tilde{f}_{R}\left(2, \omega_{R}\right)
$$

where the perturbation to the equilibrium structure caused by the shearing motion, $\tilde{f}_{R}$, satisfies the same equation as $\tilde{f},(66 a-c)$, except with a 6 in place of the 2 in front of $\tilde{f} / r^{2}$ and the boundary condition at contact is 2 in place of $\frac{1}{2}$. These changes arise because the forcing in shear flow is from the rate-of-strain tensor, and thus the perturbation to the equilibrium structure must be linear in $E$. This gives the shear flow disturbance the character of a quadrupole, with the perturbation decaying as $r^{-3}$. For diffusion, the forcing is with the wave vector $i k$, which leads to the 2 and a dipole-like solution decaying as $r^{-2}$. Also, the characteristic timescale for the relaxation of the structure in shear is the short-time self-diffusivity, and not the long-time self-diffusivity as in diffusion. This difference arises because the structure is only slightly perturbed from equilibrium in a small-amplitude shear flow, and all the particles are perturbed in the same manner, so that an individual particle need only move a small distance to relax the structure. (Note that the requirement for small departures from equilibrium, or small Péclet numbers, is $\dot{\gamma} a^{2} / D_{0}^{s}(\phi) \ll 1$, which becomes a very severe requirement on the shear rate as maximum packing is approached.) Thus, the frequency appearing in (68) is non-dimensionalized with $D_{0}^{s}(\phi) / a^{2}$.

The solution for $\tilde{f}_{R}$ at contact with the same Heaviside forcing is

$$
\begin{gathered}
\tilde{f}_{R}\left(2, \omega_{R}\right)=\frac{4}{3}\left[\frac{1+z_{R}+\frac{1}{3} z_{R}^{2}}{1+z_{R}+\frac{4}{9} z_{R}^{2}+\frac{1}{9} z_{R}^{3}}\right]\left(\pi \delta(\omega)+\frac{1}{\mathrm{i} \omega}\right), \\
z_{R}=2\left(\mathrm{i} \alpha_{R}\right)^{\frac{1}{2}}=2\left(\mathrm{i} \frac{\omega a^{2}}{2 D_{0}^{s}}\right)^{\frac{1}{2}} .
\end{gathered}
$$

where $\dagger$

The prediction for the dynamic viscosity (for both the real and imaginary parts) was shown to be in good agreement with experiment at all $\phi$ (Brady 1993b). Indeed, removing the overall amplitude of the viscosity by defining $\left(\eta_{r}(\omega)-\eta_{\infty}^{\prime}\right) /\left(\eta_{0}^{\prime}-\eta_{\infty}^{\prime}\right)$ and non-dimensionalizing frequency with $D_{0}^{s}(\phi) / a^{2}$ collapses the data for different concentrations onto a single universal curve.

Although not identical owing to the quadrupolar versus dipolar forcing, the two expressions for $\tilde{f}_{R}$ and $\tilde{f}$ at contact have the same asymptotic forms in the high- and

$\dagger$ This seems to be an appropriate place to remark that a factor of 2 was neglected in the nondimensionalization of the frequency in my earlier work on rheology (Brady 1993b). The nondimensional frequency should be $\omega a^{2} / 2 D_{0}^{s}$, not $\omega a^{2} / D_{0}^{s}$ as used earlier. 


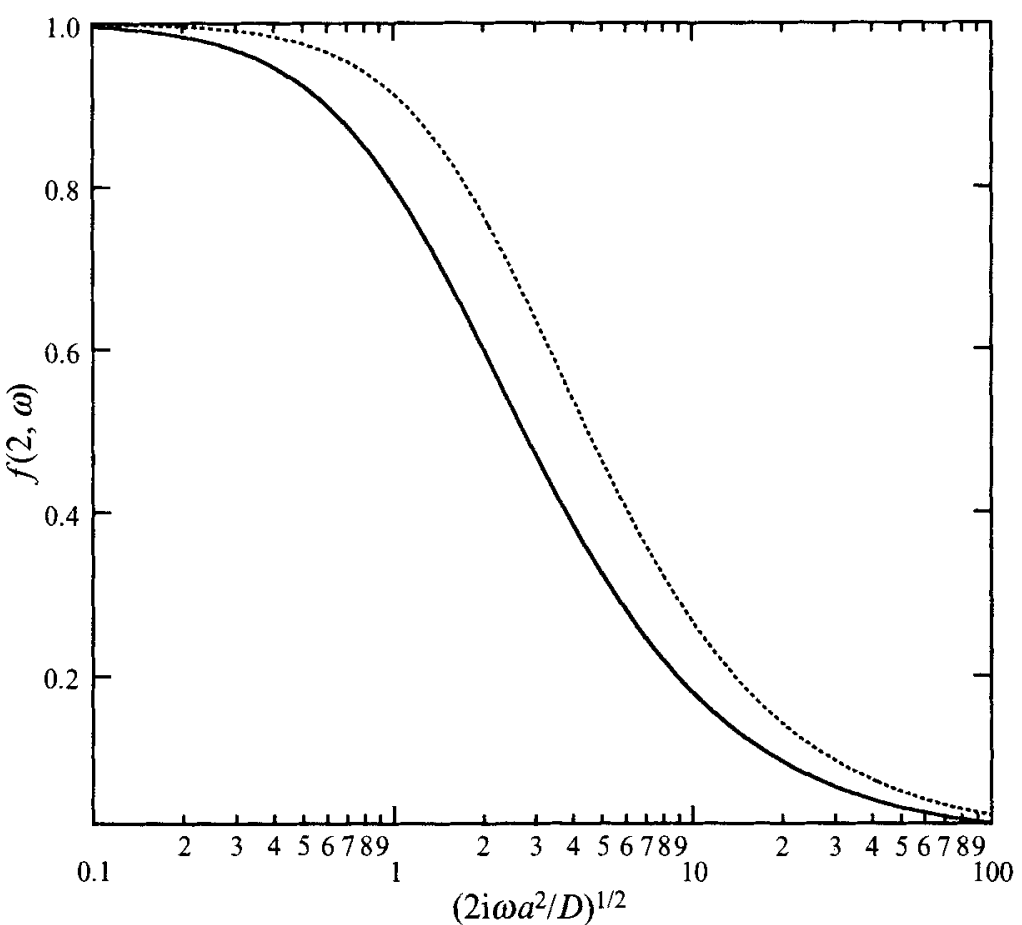

Figure 4. Comparison of the frequency response function at contact for diffusion, equation (67), solid curve, with that for viscoelastic behaviour, equation (69), dashed curve. The frequency is nondimensionalized with $a^{2}$ and the long-time self-diffusivity, $D_{\infty}^{s}(\phi)$, for the diffusive response, and the short-time self-diffusivity, $D_{0}^{s}(\phi)$, for the viscoelastic response.

low-frequency limits, behaving as $1 / z$ and $O(1)$, respectively. Here we refer to the terms in square brackets only, as the Fourier transform of the Heaviside function results from the initial condition and is not fundamental to the frequency response. In figure 4 both functions are plotted, with neither the multiplicative factors of $\frac{1}{2}$ and $\frac{4}{3}$, respectively, nor the Heaviside forcing $(\pi \delta(\omega)+1 / i \omega)$, versus their respective nondimensional frequencies. They have the same qualitative form over the entire frequency range, and thus the temporal evolution from short- to long-time diffusive behaviour should scale as the dynamic viscosity. The only important difference is that the shorttime self-diffusivity is the appropriate scaling for time for the rheological response, while it is the long-time self-diffusivity for the diffusive response. Note also, that if the overall amplitude of the diffusivities is scaled out by defining a reduced diffusivity $\left(1 / D^{s}(\omega)-1 / D_{0}^{s}\right) /\left(1 / D_{\infty}^{s}-1 / D_{0}^{s}\right)$, then this theory predicts that the reduced diffusivity should be a universal function of time non-dimensionalized by $a^{2} / D_{\infty}^{s}(\phi)$. It is also interesting to note the symbolic reciprocal relationship between the diffusivity and the viscosity: low frequency $\left(\omega \rightarrow 0\right.$ or $\left.\eta_{0}^{\prime}\right)$ corresponds to long time $\left(t \rightarrow \infty\right.$ or $\left.D_{\infty}^{s}\right)$ and vice versa.

The frequency response predicted by (67) gives the following temporal behaviour: as $t \rightarrow \infty$ the approach to the long-time self-diffusivity decays as $t^{-\frac{3}{2}}$, scaled, of course, with $a^{2} / D_{\infty}^{s}$, which should be experimentally measurable. Indeed, the numerical simulations of Cichocki \& Hinsen (1990) show precisely this behaviour. For short times, the temporal response is predicted to rise from zero as $t^{\frac{1}{2}}$, coming from the $\omega^{-\frac{1}{2}}$ decay in the term in square brackets in $\tilde{f}(2, \omega)$. This frequency response decaying as $\omega^{-\frac{1}{2}}$ as $\omega \rightarrow \infty$ 
is too slow and gives an incorrect prediction for the short-time response. This slow decay can be traced directly to the neglect of hydrodynamic interactions in the dilute pair-evolution equation used to estimate $\tilde{f}$. Retaining hydrodynamics will give $\tilde{f} \sim 1 / \alpha$ as $\omega \rightarrow \infty$, which is a more rapid frequency decay, corresponding to a temporal response proportional $t$. Exactly the same behaviour occurs in the viscosity problem, and the resolution of this slow decay is explained more fully in Brady (1993b).

\section{Conclusions}

In this paper we have analysed the temporal decay of density fluctuations and through this obtained expressions for both the short- and long-time self-diffusivities in concentrated colloidal dispersions. The short-time self-diffusivity was shown to be the equilibrium average mobility of a tracer particle and thus fundamentally a hydrodynamic quantity. The long-time self-diffusivity, on the other hand, requires the tracer to wander far from its starting point and in so doing deform the suspension structure. It was argued that the deformation and relaxation of the structure must be determined self-consistently using the long-time self-diffusivity. This led to the prediction that the long-time self-diffusivity was given by the product of the hydrodynamically determined short-time self-diffusivity, $D_{0}^{s}$, and a thermodynamically controlled structural quantity $[1+2 \phi g(2 ; \phi)]^{-1}$.

An alternative and simple physical picture of the long-time self-diffusivity can be argued through the Stokes-Einstein relation that states that the diffusivity is proportional to the mobility of an individual particle divided by its characteristic size. As a tracer particle moves it carries along its neighbours or cage, and thus its effective size is increased and as a result its motion slowed down. This increase in size is a 'geometric' problem, occurring whether or not there are hydrodynamic interactions among particles. Thus, the slowing down due to the increased size is controlled by structure and is directly proportional to the radial-distribution function at contact, $g(2)$. The mobility of an individual particle is simply the short-time self-diffusivity, and we arrive at the scaling prediction for the long-time self-diffusivity, $D_{\infty}^{s}(\phi) \sim$ $D_{0}^{s}(\phi) / g(2 ; \phi)$.

The prediction of this theory for the long-time self-diffusivity of Brownian hard spheres, $D_{\infty}^{s}(\phi)=D_{0}^{s}(\phi)[1+2 \phi g(2 ; \phi)]^{-1}$, shows that as maximum packing is approached the tracer's motion ceases according to $D_{\infty}^{s} \sim 0.562 D_{0}\left(1-\phi / \phi_{m}\right)^{2}$ as $\phi \rightarrow \phi_{m}$; one power of $\left(1-\phi / \phi_{m}\right)$ coming from the vanishing of the short-time self-diffusivity and the other from the singular behaviour of the radial-distribution function at contact. As shown in figure 2 this prediction is in excellent accord with experiment over the entire range of volume fractions.

For particles that interact through hard-sphere-like repulsive interparticle forces, the same formula applies. The only change is that the short-time self-diffusivity is set by the true or hydrodynamic volume fraction $\phi$, but the radial-distribution function is set by the 'thermodynamic' volume fraction - the volume fraction that characterizes the equilibrium structure. For repulsive forces that have roughly the same range as the actual particle radius, the predicted long-time self-diffusivities will be virtually identical to those of Brownian hard spheres. This is because $D_{0}^{s}$ decreases linearly with volume fraction (nearly so for almost all $\phi$ and precisely near $\phi_{m}$ ), so that the slowing down due to mobility and structure will both contribute. This fact must explain why the experimental results, which are for real colloidal particles that are approximately hard spheres, are all in agreement - the behaviour is not sensitive to any short-range repulsive forces. 
For long-range hard-sphere-like repulsive forces, such that the equilibrium structure is set by and reaches its maximum thermodynamic volume fraction, $\phi_{b m}$, where the length $b$ characterizes the range of the repulsive forces, long before the true or hydrodynamic volume fraction is near maximum packing, the theory predicts that the long-time self-diffusivity slows down due only to the increase in size of the tracer, i.e. $D_{\infty}^{s} \sim D_{0}\left[1+2 \phi_{b} g\left(2, \phi_{b}\right)\right]^{-1}$. Thus, $D_{\infty}^{s} \sim D_{0}\left(1-\phi_{b} / \phi_{b m}\right)$ as $\phi_{b} \rightarrow \phi_{b m}$. The individual particle mobility is simply the infinite-dilution Stokes-Einstein diffusivity $D_{\mathbf{0}}$.

Rallison (1988) has developed a theory for the long-time self-diffusivity in concentrated suspensions that seems to have the same physical notion that a particle is slowed down by carrying along its neighbours. From a consideration of a onedimensional random walk of sterically interacting particles, he argues that the longtime self-diffusivity is given by the individual particle diffusivity divided by the number of neighbours a particle carries. Rallison then develops a heuristic scheme to estimate the size of the region of influence of the moving tracer as a function of time, i.e. the number of neighbours being carried, and from this obtains an estimate for the longtime self-diffusivity. Physically this is similar to the idea put forth here, and through our calculation of the perturbation to the equilibrium structure we have a direct determination of the number of carried neighbours. Rallison did not consider hydrodynamic interactions, and when he applied his theory to a concentrated suspension of spherical particles, he predicted that the long-time self-diffusivity would vanish quadratically at random close packing. This prediction contradicts the results obtained here, for we have argued that in the absence of hydrodynamic interactions the long-time self-diffusivity should vanish linearly at random close packing (cf. (52)). The numerical calculations of Cichocki \& Hinsen $(1990,1992)$ in figure 1 also show that in the absence of hydrodynamic interactions the long-time self-diffusivity vanishes much more slowly than quadratically.

We have also shown that the self-diffusivities are inversely proportional to the suspension viscosities. The short-time self-diffusivity scales as $k T / \eta a \eta_{\infty}^{\prime}$, with $\eta_{\infty}^{\prime}$ the high-frequency dynamic viscosity, while the long-time self-diffusivity scales as $k T / \eta a \eta_{0}^{\prime}$, with $\eta_{0}^{\prime}$ the zero-frequency or steady-shear viscosity. This inverse relationship between the self-diffusivity and the suspension viscosity carries over to arbitrary frequencies if one defines a frequency-dependent self-diffusivity $D^{s}(\omega)$. Indeed, it was shown that the normalized frequency-dependent self-diffusivity $\left(1 / D^{s}(\omega)-1 / D_{0}^{s}\right) /\left(1 / D_{\infty}^{s}-1 / D_{0}^{s}\right)$ should be a universal function for all volume fractions of the non-dimensional frequency $\omega a^{2} / D_{\infty}^{s}(\phi)$, and that this frequency dependence should be virtually identical to that of the reduced viscosity $\left(\eta_{r}(\omega)-\eta_{\infty}^{\prime}\right) /\left(\eta_{0}^{\prime}-\eta_{\infty}^{\prime}\right)$, when the latter's frequency is normalized with the short-time self-diffusivity, $D_{0}^{s}(\phi) / a^{2}$. This universal scaling for the frequency-dependent viscosity has been verified experimentally (van der Werff et al. 1989), and we put forth this prediction for the self-diffusivity to be tested experimentally. The test need not be done in the frequency domain, but can be done simply by comparing the temporal evolution from the short- to long-time selfdiffusivity with the temporal increase in viscosity in the start up of steady shear.

The theory we have put forward for determining the long-time self-diffusivity forms the natural starting point for further studies. We have considered the simplest case of hard-sphere-like particles, and for ease of analytical calculation estimated $f_{2}$ at contact ignoring the spatial variation of the equilibrium structure, but both of these restrictions can be relaxed. It is a straightforward matter to solve the pair-evolution equation numerically for a specific form of the interparticle potential, i.e. $g(r)$, and a variety of three-particle closures can be used. Indeed, knowing that the hard-sphere suspension undergoes a phase transition to a solid at $\phi \approx 0.5$, it would be interesting to see what 
effect this structural change has on the long-time self-diffusivity. Perhaps the seemingly more rapid drop in the experimental long-time self-diffusivities near $\phi \approx 0.5$ than predicted by the present theory could be explained by this phase transition.

The theory can also naturally be extended to consider the diffusive behaviour away from equilibrium, for example in shearing flow (Qui et al. 1988). The only modifications needed are to add the convective motion $U P_{N}$ to the flux of a particle, which influences both the expression for the time derivative of the self-intermediate scattering function and the pair-evolution equation. The latter now becomes a convection-diffusion equation with an appropriate Péclet number measuring the relative importance of convection to diffusion. From the literature on convection-diffusion problems one can immediately recognize that in simple shearing motion the effect of flow will enhance the self-diffusivity analogous to the dispersion studied by Taylor (1953). It should also be possible to use this formulation to study the diffusive motion far from equilibrium where convection dominates and the diffusivity scales hydrodynamically as $\dot{\gamma} a^{2}$.

The factorization of the long-time self-diffusivity into the hydrodynamically determined short-time self-diffusivity and a geometrically or thermodynamically determined structural term also carries over to other situations. Specifically, it would be interesting to see how this factorization works for the translational and rotational diffusivities in rod-like suspensions or for the long-time self-diffusivity in fixed arrays. Each of these problems can be formulated in a very similar manner and recent work on diffusion in fixed arrays shows that accurate predictions can be obtained by this factorization (Brady 1994).

This work was supported in part by Grant No. CTS-9020646 from the National Science Foundation.

\section{REFERENCES}

ACKerson, B. J. 1978 Correlations for interacting Brownian particles. II. J. Chem. Phys. 69, 684. BATCHELOR, G. K. 1983 Diffusion in a dilute polydisperse system of interacting spheres. J. Fluid Mech. 131, 155 and Corrigendum J. Fluid Mech. 137, 1983, 467.

BEENAKKER, C. W. J. 1984 The effective viscosity of a concentrated suspension of spheres (and its relation to diffusion). Physica A 128, 48.

BeENAKKER, C. W. J. \& MAZUR, P. 1984 Diffusion of spheres in a concentrated dispersion II. Physica A 126, 349.

Berne, B. J. \& Pecora, R. 1976 Dynamic Light Scattering. Wiley.

Blaaderen, A., van, Peetermans, J., Maret, G. \& Dhont, J. K. G. 1992 Long-time self diffusion of spherical colloidal particles measured with fluorescence recovery after photobleaching. $J$. Chem. Phys. 96, 4591.

Bossis, G., BRADY, J. F. \& Mathis, C. 1988 Shear-induced structure in colloidal suspensions. I. Numerical simulations. J. Colloid Interface Sci. 126, 1.

Brady, J. F. 1993 a Brownian motion, hydrodynamics and the osmotic pressure. J. Chem. Phys. 98, 3335.

BRADY, J. F. $1993 b$ The rheological behavior of concentrated colloidal dispersions. J. Chem. Phys. $99,567$.

BRADY, J. F. 1994 Hindered diffusion in porous media. $J$. Colloid Interface Sci. (to be submitted).

Carnahan, N. F. \& Starling, K. E. 1969 Equation of state for nonattracting rigid spheres. $J$. Chem. Phys. 51, 635.

Сiсноскі, B. \& Felderhof, B. U. 1992 Time-dependent self-diffusion in a semidilute suspension of Brownian particles. J. Chem. Phys. 96, 4669.

CiCHOCKI, B. \& HinsEn, K. 1990 Self and collective diffusion coefficients of hard sphere suspensions. Ber. Bunsenges. Phys. Chem. 94, 243. 
CICHOCKI, B. \& HINSEN, K. 1992 Dynamic computer simulation of concentrated hard sphere suspensions. Physica A 187, 145.

Cohen, E. G. D. \& SChepper, I. M. DE 1991 Note on transport processes in dense colloidal suspensions. J. Statist. Phys. 63, 241.

Hess, W. \& Klein, R. 1983 Generalized hydrodynamics of systems of Brownian particles. Adv. Phys. 32, 173.

Kops-Werkhoven, M. M. \& Fijnaut, H. M. 1982 Dynamic behavior of silica dispersions studied near the optical matching point. J. Chem. Phys. 77, 2242.

LADD, A. J. C. 1990 Hydrodynamic transport coefficients of random dispersions of hard spheres. $J$. Chem. Phys. 93, 3483.

Leegwater, J. A. \& Szamel, G. 1992 Dynamical properties of hard-sphere suspensions. Phys. Rev. A 46, 4999.

Medina-Noyola, M. 1988 Long-time self-diffusion in concentrated colloidal dispersion. Phys. Rev. Lett. 60, 2705.

Megen, W. VAN, Underwood, S. M. \& SNOOK, I. 1986 Tracer diffusion in concentrated colloidal dispersions. J. Chem. Phys. 85, 4065.

MegEN, W. van \& UNDERWOOD, S. M. 1989 Tracer diffusion in concentrated colloidal dispersions. III. Mean squared displacements and self-diffusion coefficients. J. Chem. Phys. 91, 552.

Ottewill, R. H. \& Williams, N. St. J. 1987 Study of particle motion in concentrated dispersions by tracer diffusion. Nature 325, 232.

Phillips, R. J., BRAdY, J. F. \& Bossis, G. 1988 Hydrodynamic transport properties of hard-sphere dispersions. I. Suspensions of freely mobile particles. Phys. Fluids 31, 3462.

Phung, T. N. 1993 Behavior of concentrated colloidal dispersions by Stokesian dynamics simulation. PhD thesis, California Institute of Technology.

PusEy, P. N. 1991 Colloidal suspensions. In Liquids, Freezing and Glass Transition (ed. J. P. Hansen, D. Levesque \& J. Zinn-Justin). Elsevier.

PUSEY, P. N. \& MEgEN, W. VAN 1983 Measurement of the short-time self-mobility of particles in concentrated suspension. Evidence for many-particle hydrodynamic interactions. J. Phys. Paris 44, 285.

Qui, X., Ou-Yang, H. D., Pine, D. J. \& Chaikin, P. M. 1988 Self-diffusion of interacting colloids far from equilibrium. Phys. Rev. Lett. 61, 2554.

Rallison, J. M. 1988 Brownian diffusion in concentrated suspensions of interacting particles. $J$. Fluid Mech. 186, 471.

Rallison, J. M. \& Hinch, E. J. 1986 The effect of particle interactions on dynamic light scattering from a dilute suspension. J. Fluid Mech. 167, 131.

Russel, W. B. \& Glendinning, A. B. 1981 The effective diffusion coefficient detected by dynamic light scattering. J. Chem. Phys. 74, 948.

Selim, M. S, Al-NAafa, M. A. \& Jones, M. C. 1993 Brownian diffusion of hard spheres at finite concentrations. AIChE J. 39, 3.

Szamel, G. \& LeEgWateR, J. A. 1992 Long-time self-diffusion coefficients of suspensions. Phys. Rev. A 46, 5012.

TAYLOR, G. I. 1953 Dispersion of soluble matter in solvent flowing slowly through a tube. Proc, $R$. Soc. Lond. A 219, 186.

Veluwen, A. van \& LekKerkerker, H. N. W. 1988 NonGaussian behavior of the displacement statistics of interacting colloidal particles. Phys. Rev. A 38, 3758.

WerfF, J. C. van DeR, Kruif, C. G. DE, Blom, C. \& Mellema, J. 1989 Linear viscoelastic behavior of dense hard-sphere dispersion. Phys. Rev. A 39, 418.

Woodcock, L. V. 1981 Glass transition in the hard sphere model and Kauzman's paradox. Ann. NY Acad. Sci. 37, 274. 